\title{
Quantification of mid and late evoked sinks in laminar current source density profiles of columns in the primary auditory cortex
}

\author{
Markus K. Schaefer *, Julio C. Hechavarría and Manfred Kössl \\ Institute for Cell Biology and Neuroscience, AK Neurobiology and Biosensors, Goethe University, Frankfurt/Main, Germany
}

Current source density (CSD) analysis assesses spatiotemporal synaptic activations at somatic and/or dendritic levels in the form of depolarizing current sinks. Whereas many studies have focused on the short (<50 ms) latency sinks, associated with thalamocortical projections, sinks with longer latencies have received less attention. Here, we analyzed laminar CSD patterns for the first $600 \mathrm{~ms}$ after stimulus onset in the primary auditory cortex of Mongolian gerbils. By applying an algorithm for contour calculation, three distinct mid and four late evoked sinks were identified in layers I, III, Va,

OPEN ACCESS

Edited by:

Manuel S. Malmierca,

University of Salamanca, Spain

Reviewed by: Jagmeet S. Kanwal, Georgetown University, USA Matthias Deliano, Leibniz Institute for Neurobiology,

Germany

${ }^{*}$ Correspondence:

Markus K. Schaefer,

Institute for Cell Biology and Neuroscience, AK Neurobiology and

Biosensors, Goethe University,

Max-von-Laue-Straße 13, 60438 Frankfurt/Main, Germany markus.sbio@web.de

Received: 18 June 2015 Accepted: 14 September 2015 Published: 02 October 2015

Citation: Schaefer MK, Hechavarría JC and Köss/ M (2015) Quantification of mid and late evoked sinks in laminar current source density profiles of columns in the primary auditory

cortex.

Front. Neural Circuits 9:52 doi: 10.3389/fncir.2015.00052
Vla, and VIb. Our results further showed that the patterns of intracortical informationflow remained qualitatively similar for low and for high sound pressure level stimuli at the characteristic frequency (CF) as well as for stimuli \pm 1 octave from CF. There were, however, differences associated with the strength, vertical extent, onset latency, and duration of the sinks for the four stimulation paradigms used. Stimuli one octave above the most sensitive frequency evoked a new, and quite reliable, sink in layer Va whereas low level stimulation led to the disappearance of the layer Vlb sink. These data indicate the presence of input sources specifically activated in response to level and/or frequency parameters. Furthermore, spectral integration above vs. below the CF of neurons is asymmetric as illustrated by CSD profiles. These results are important because synaptic feedback associated with mid and late sinks-beginning at $50 \mathrm{~ms}$ post stimulus latency -is likely crucial for response modulation resulting from higher order processes like memory, learning or cognitive control.

Keywords: local field potentials, current source density, late evoked potentials, intracortical network processing, spectral integration, horizontal projections, cortical layers, primary auditory cortex

\section{Introduction}

Population activity of neurons in the mammalian cortex and their interaction with subcortical as well as with other cortical regions can be evaluated using local field potentials (LFP). These summed potentials are thought to reflect synaptic activity and the sequence of characteristic LFP waves is considered to indicate distinct stages in the cortical processing of information (Barth and Di, 1991; Medvedev and Kanwal, 2004). Studies on the differential effects of anesthesia (Barth and Di, 1990), sleep (Knight et al., 1985), and alertness (Satya-Murti et al., 1983; Knight et al., 1985) on the amplitudes and spatiotemporal distribution of LFP peaks, 
gave evidence that these potentials may be generated by multiple brain regions. In the auditory cortex, the early positive/negative biphasic wave (P1/N1), which is commonly found in rats (Barth and Di, 1990; Simpson and Knight, 1993; Takahashi et al., 2007), guinea pigs (Kraus et al., 1985), gerbils (Ohl et al., 2000), and cats (Kaga et al., 1980), most likely reflects depolarization triggered by thalamic or intracortical inputs (Kaga et al., 1980; Barth and Di, 1990; Di and Barth, 1992; Thomas et al., 1993; Ohl et al., 2000; Kaur et al., 2004).

Successive waves are proposed to be generated by different areas of the primary and secondary cortical fields, the frontal cortex (FC), the centro-parietal cortex, association cortex or hippocampus (Picton et al., 1974; Skrebitsky and Sharonova, 1976; Kraus et al., 1985; Näätänen and Picton, 1987; Barth and Di, 1990; Santos Filha and Matas, 2010).

However, single LFP recordings representing the sum of inhibitory and excitatory synaptic activity cannot provide precise spatial information about a layer- or depth-specific location of various activation components, although they have been reported to induce lower thresholds for responses to tone bursts than for simultaneously recorded spiking activity (Galvan et al., 2002). The current source density (CSD) analysis, which is based on the second spatial derivative of the field potentials along the radial depth, localizes synaptic inputs. This method provides precise spatial and temporal information about the functional weights of synaptic activity (sinks) and therefore points to the mechanism of their generation. It thus allows to trace the neuronal information flow (Nicholson and Freeman, 1975; Mitzdorf, 1985).

Studies using CSD analysis in combination with cortical silencing showed that initial sinks originate from thalamic or intracortical regions (Kaur et al., 2005; Happel et al., 2010). The subsequent sinks were thus hypothesized to be evoked through transynaptic intracortical processing. Repetitive after-discharges originating from the thalamus or inputs from outside of the AC such as the FC, the contralateral hemisphere or the hippocampus are also possible generators (Rappelsberger et al., 1981; Mitzdorf, 1985). Mid and late sinks could be crucial for understanding higher order processes like memory, learning or cognitive control and provide the basis for modeling precise neuronal circuits.

Several studies on early and mid sinks proposed that intracortical connections are responsible for spectrally distant inputs (Kaur et al., 2004, 2005; Tomioka et al., 2005; Kurt et al., 2008; Happel et al., 2010; Moeller et al., 2010). In contrast, low level sounds are believed being temporally integrated by the pressure envelope of the sound (Heil and Neubauer, 2001, 2003; Heil et al., 2008). It would be interesting to compare both integration mechanisms at the cortical level. To our knowledge, studies on level and spectral integration of late sinks have not yet been done. Differences in these sinks evoked by stimuli at the borders of receptive fields could be a result of stimulusspecific proportioned spatial and temporal interactions and convergences and thus help to complete the understanding of these integration mechanisms.

To study the differences, origins, laminar locations and temporal characteristics of sinks, we qualitatively and quantitatively characterized the mid and late evoked current sinks (50-575 ms post stimulus) evoked by stimuli at the borders of receptive fields in the primary auditory cortex of Mongolian gerbils. We could identify three mid and four late evoked sinks, which were less reliably evoked compared to the initial two sinks in layers III/IV and V/VI. The high resolution of laminar profiles allowed us to propose a hypothetical scheme of the specific sink generators based on results of previous studies and on the cortical architecture. Although the intracortical information-flow patterns remained qualitatively similar for the stimuli, we could find differences in the strength, vertical extent, onset latency, and duration of the sinks, which could be caused by either temporal or spectral integration. The appearance of a new sink emerged in layer $\mathrm{Va}$ when a stimulus one octave above the characteristic frequency (CF) was tested and the disappearance of the layer VIb sink at low level stimulation indicated the presence of input sources specifically activated in dependence of level and/or frequency. Our results further indicated an asymmetry regarding synaptic inputs associated with low vs. high frequency processing in the cortex.

\section{Materials and Methods}

The study was carried out in 8 adult Mongolian gerbils (Meriones unguiculatus; age: 6-10 month; body weight: 59-79 g) of both sexes (five females and three males). Animals were taken from a breeding colony in the Institute for Cell Biology and Neuroscience, Goethe University, Frankfurt am Main, Germany. All experiments were conducted in accordance with the regulations by the International National Institutes of Health Guidelines for Animals in Research and with ethical standards for the care and use of animals in research defined by German Law for the protection of experimental animals (Experimental permit \# F104/60).

\section{Surgical Procedures}

All animals were initially anesthetized with a mixture of ketamine (100 mg/kg; Ketavet, Pfizer, New York, USA), xylazine (20 mg/kg; Rompun, 2\%; BayerVital, Berlin, Germany) and isotonic sodium chloride solution $(100 \mathrm{mg} / \mathrm{kg}$; 0.9\%; B. Braun, Melsungen, Germany). Anesthesia was maintained with the same mixture throughout the whole experiment with an injection pump (flow-rate: $75 \mathrm{mg} / \mathrm{kg} / \mathrm{h}$, Genie, Kent Scientific Corporation, Torrington, CT, USA) while regularly monitoring the hindlimb withdrawal reflex and whisker activity. Body temperature was kept at $37^{\circ} \mathrm{C}$ using a thermostatic heating blanket. After removing the musculus temporalis, the skull was cleaned and a custom-made metal rod $(1 \mathrm{~cm}$ length, $0.3 \mathrm{~cm}$ diameter) was glued onto it using dental cement (Paladur; Heraeus Kulzer, Hanau, Germany). A craniotomy $(\sim 3 \times 3 \mathrm{~mm})$ was made using a drilling device to expose the left auditory cortex. The opening was cleaned from bone splints and the dura mater was completely removed with an injection needle.

\section{Electrophysiological Recordings}

Recordings were performed in a custom-built sound-proof and electrically-shielded chamber. The neuronal activity was recorded using commercially available linear probes 
(Model: A1 × 16-3 mm-100-177-A16, NeuroNexus, Ann Arbor, MI, USA) with 16 contacts (impedance: $0.5-3 \mathrm{M} \Omega$; spacing: $100 \mu \mathrm{m}$ ) spanning $1500 \mu \mathrm{m}$. Such electrode design is well suited for obtaining recordings from entire cortical columns, which span $\sim 1300 \mu \mathrm{m}$ in the Mongolian gerbil (Sugimoto et al., 1997). Using a micro-manipulator system (PM 10/1, Science Products $\mathrm{GmbH}$, Hofheim, Germany), electrodes were inserted slowly into the brain $(20 \mu \mathrm{m} / \mathrm{s})$ and placed perpendicular to the pial surface at a depth of $\sim 1500 \mu \mathrm{m}$, so that the top channel was located above the cortical surface. Orthogonality between electrode and surface was controlled by adjusting the animal's head several times until the altitude differences between the four corners of the temporal hole were $<70 \mu \mathrm{m}$ utilizing the electrode as an altimeter. Using this technique once for each animal, the characteristic frequencies of neurons recorded at 400-1000 $\mu \mathrm{m}$ (layers IV-VIa) of each track (6.4 \pm 3.9 tracks per animal) were constant within a range of \pm 0.34 octaves.

Layer depth localization was adopted from a previous study by Sugimoto et al. (1997): layer I was located at 0-120 $\mu \mathrm{m}$, layer II at $120-210 \mu \mathrm{m}$, layer III at $210-410 \mu \mathrm{m}$, layer IV at $410-560 \mu \mathrm{m}$, layer $\mathrm{V}$ at $560-850 \mu \mathrm{m}$, and layer VI at $850-1300 \mu \mathrm{m}$ depth from the cortical surface. Neuronal activity was preamplified (10×, $\mu$ PA16, Multichannel Systems, Reutlingen, Germany) and recorded using a multichannel recording system (amplification: $1000 \times$, ME32, Multichannel Systems, Reutlingen, Germany). To obtain LFP, recorded signals were digitally bandpass-filtered offline between 0.2 and $300 \mathrm{~Hz}$ (butterworth, 2nd order) and notch-filtered at $50 \mathrm{~Hz}$ to remove humming noise, downsampled from 50 to $20 \mathrm{kHz}$ and stored in a computer for further analysis.

\section{Acoustic Stimulation}

Pure tones were digitally synthesized and controlled using a custom-written program in Matlab (R2007b, MathWorks, Natick, MA, USA). Stimuli were generated by an external soundcard (e18 dac, exaSound, Toronto, Canada, sampling rate: $192 \mathrm{kHz}$ ), amplified (RB-1050, Rotel Electronics, Tokyo, Japan) and delivered from a calibrated speaker (SS-MS835, Sony, Tokyo, Japan). The calibration curve was obtained with a Brüel and Kjaer sound recording system ( $\frac{1}{4}$-inch Microphone 4135, Microphone Preamplifier 2670, Brüel and Kjaer, Naerum, Denmark) connected to a conditioning microphone amplifier (Nexus 2690, Brüel and Kjaer, Naerum, Denmark). During the experiment, the speaker was placed in front of the animal's right ear at a distance of $20 \mathrm{~cm}$. We presented the animal with a pseudorandomized series of pure tones at different intensities (step size: $10 \mathrm{~dB}$, range: $0-80 \mathrm{~dB}$ SPL) with either a logarithmic or linear frequency spacing (step size: 0.5 oct $/ 3.5$ $\mathrm{kHz}$, range: $0.25-64 \mathrm{kHz} / 0.5-56.5 \mathrm{kHz}$ ), depending on the respective neuronal receptive field (borderline at $11 \mathrm{kHz}$ ). We calculated neuronal tuning curves for a threshold value of $30 \%$ of the maximum spiking rate. The $\mathrm{CF}$ was defined as the stimulus frequency that elicited a response at the minimum threshold (MT) of the tuning curve. The CF was calculated from the neuronal response obtained in layer V/VI, and for the present data set only penetrations that yielded sensitive (MT $<50 \mathrm{~dB}$ SPL) and V-shaped tuning curves in accordance with the tonotopic map of gerbils (Thomas et al., 1993) were considered. The response to four stimuli combinations was used for analyzing columnar CSD patterns: (1) pure tone at CF at 80 $\mathrm{dB}$ SPL (from now on CF80); (2) pure tone one octave below the CF at $80 \mathrm{~dB}$ SPL (from now on -1oct); (3) pure tone one octave above the CF at $80 \mathrm{~dB}$ SPL (from now on +1 oct); and (4) pure tone at the $\mathrm{CF} \leq 24 \mathrm{~dB}$ above the individual MT (from now on $\mathrm{CF} 24+$ ). The level of the latter was conditioned by the measuring level step size of $24 \mathrm{~dB}$. All stimuli were presented in pseudorandomized order and sampled at $192 \mathrm{kHz}$. The duration was $30 \mathrm{~ms}$ with a $5 \mathrm{~ms}$ rise-fall. The interstimulus interval was set at $0.6 \mathrm{~s}$.

\section{Current Source Density Analysis}

Neuronal activity was recorded simultaneously from all cortical layers of 51 penetration sites in the left primary auditory cortex. Bandpass-filtered LFPs were averaged over 25 stimulus repetitions (Figure 1A). The standard CSD method assumes a homogeneous activity along the horizontal direction and uses a discretized version of the Poisson's equation. It further assumes that the extracellular medium acts as a volume conductor that is ohmic at the relevant frequency range (Nicholson and Freeman, 1975; Mitzdorf, 1985; Pettersen et al., 2006; Szymanski et al., 2009). We calculated one-dimensional CSD profiles from the second spatial derivative of the LFP, which can be approximated using the following formula:

$$
\frac{\delta^{2} \phi}{\delta \mathrm{z}^{2}} \approx \frac{\phi\left(\mathrm{z}_{0}+\mathrm{n} \Delta \mathrm{z}\right)+\phi\left(\mathrm{z}_{0}-\mathrm{n} \Delta \mathrm{z}\right)-2 \phi\left(\mathrm{z}_{0}\right)}{(\mathrm{n} \Delta \mathrm{z})^{2}}
$$

The double of the field potential $(\phi)$ at the cortical depth $z_{0}$ is subtracted from the summated adjacent field potentials above $\left(\mathrm{z}_{0}+\mathrm{n} \Delta \mathrm{z}\right)$ and below $\left(\mathrm{z}_{0}-\mathrm{n} \Delta \mathrm{z}\right)$ the field potentials at depth $\mathrm{z}_{0}$ (interchannel distance $\Delta \mathrm{z}=100 \mu \mathrm{m}$ ) and divided by the differentiation grid $(\mathrm{n} \Delta \mathrm{z} ; n=1)$. For the CSD calculation, a modified version of the iCSDplotter toolbox was used (Pettersen et al., 2006). Estimates for the CSD at top and bottom electrode channels were provided by the method of Vaknin et al. (1988). To reduce spatial noise, a three-point Hamming filter was applied (Rappelsberger et al., 1981):

$$
\phi_{\text {filt }}(\mathrm{z})=0.23 \phi\left(\mathrm{z}_{0}+\mathrm{n} \Delta \mathrm{z}\right)+0.23 \phi\left(\mathrm{z}_{0}-\mathrm{n} \Delta \mathrm{z}\right)+0.54 \phi\left(\mathrm{z}_{0}\right)
$$

In the resulting laminar CSD profiles (Figure 1B), current sinks are classically interpreted to indicate excitatory events e.g., axonal depolarizations and excitatory or inhibitory synaptic activations and current sources are in most cases the passive return currents (Mitzdorf, 1985). Visualization of laminar profiles was improved by linear channel interpolation.

\section{Contour Calculation and Parameter Quantification of Sinks}

Accurately determining the onset and offset latencies of sinks has been described as difficult in previous studies (Kaur et al., 2004; Happel et al., 2010). Here, to obtain global criteria for separating individual sinks, we used a two step calculation method. In the first step, contours were plotted around all sinks of a laminar profile, which surpassed $8 \%$ of the maximum sink amplitude of the CSD profile (Figure 1C) elicited with CF80 


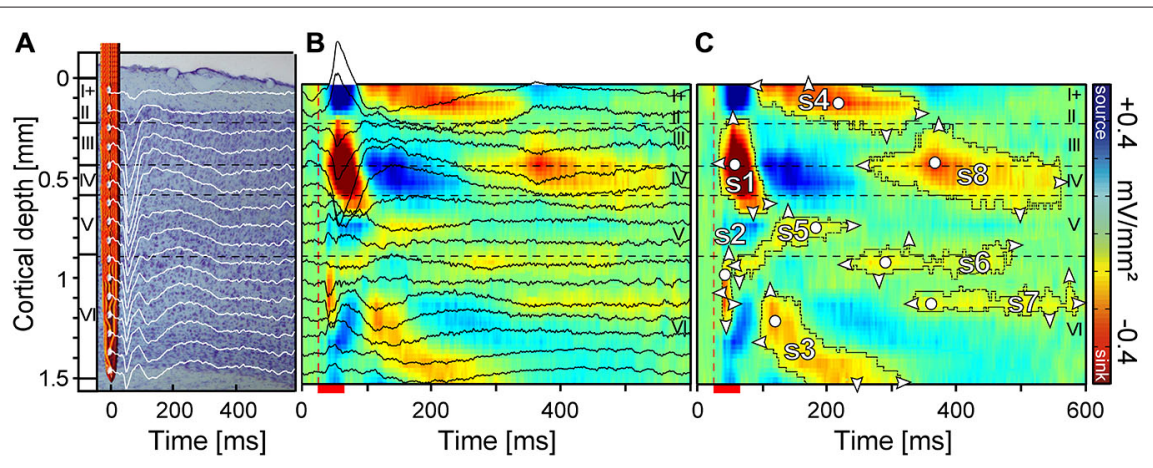

FIGURE 1 | Laminar local field potentials (LFP) and current source density (CSD) profile of a primary auditory cortex site tuned to 5.7 kHz. (A) Field potentials were recorded with a linear-array multicontact electrode covering all six cortical layers. Recordings were obtained simultaneously at each depth at an interchannel distance of $100 \mu \mathrm{m}$. (B) CSD profiles were calculated from field potentials with color indicating current strength and direction. Current sinks (red) are classically interpreted as net inward transmembrane currents and current sources (blue) are net outward currents. (C) For the quantification of sinks, several contour lines were plotted using a criterion of $8 \%$ of the maximum sink strength. Eight sinks (s1-s8) could be defined within a time window of 600 ms. White circles represent the location of the maximum strength and white triangles the horizontal and vertical dimensions. Horizontal dashed lines indicate layer borders while the vertical dashed line marks the beginning of pure tone stimulation. Red scale bar represents pure tone stimulus duration of 30 ms.

stimulation of the respective column. Contours were calculated using Matlab's contour function. In the second step, contour depending parameters of sinks such as area size, onset latency, duration, vertical extent, maximum strength and its depth were automatically calculated with custom-written programs using Matlab. Resulting sink patches were merged together if the temporal distance accounted for less than $25 \mathrm{~ms}$. Fusions between sinks were manually separated at their narrowest point in accordance with the sink structure of the averaged CSD patterns. In the result section we focus on $\mathrm{s} 1, \mathrm{~s} 3, \mathrm{~s} 5$, and $\mathrm{s} 8$ as we assumed that analyzing those sinks could be sufficient to quantitatively access the stimulus-specific characteristics of sinks and layer dependent differences. In our analysis, s3 was of special interest, as its activation spanned over a large area within layer VI likely involving different types of pyramidal cells projecting to thalamic nuclei. Sink s5 was chosen, despite of its small area of activation, as it was reliably evoked and it is the only sink in layer $\mathrm{V}$ which is known to receive input from subcortical (medial division of the geniculate body) and contralateral areas such as the frontal, entorhinal and auditory cortex (Linden and Schreiner, 2003). Sink s8 had the largest area compared to all remaining sinks and its location in the main thalamic input layers III/IV suggests an important role for the integration of ispilateral and contralateral inputs (Linden and Schreiner, 2003).

\section{Statistical Analysis}

Data of sink parameters were tested for normal distribution with a Kolmogorov-Smirnov test. A non-parametric one-way analysis of variance (Kruskal-Wallis) was then applied in combination with a multiple comparison post hoc test in Matlab (multcompare function). All statistical analysis was performed with custom-written programs using Matlab. Tests that rendered $p$ values $<0.05$ were considered as significant.

\section{Recording Site and Map Construction}

The location and size of the gerbil's AC is schematically represented in Figure 2A. Several functional areas (Figure 2B) were identified in previous studies (Thomas et al., 1993; Budinger et al., 2000, 2008). All recording locations (Figure 2B) were verified by using the stereotaxic coordinates of penetration points and the suture intersection of parietal, sphenoidal and temporal bone as reference. The course of blood vessels was variable and therefore it could not be used for localization of penetrations. We chose the recording sites to obtain an evenly distributed representation of neurons in the AI at the planar and laminar level. CFs of the studied recording points ranged between 0.5 and $28 \mathrm{kHz}$, with 24 penetrations above $10 \mathrm{kHz}$ and 27 below this value.

\section{Results}

LFPs were measured along 51 penetrations in the left primary auditory cortex (AI) of eight adult Mongolian gerbils, while

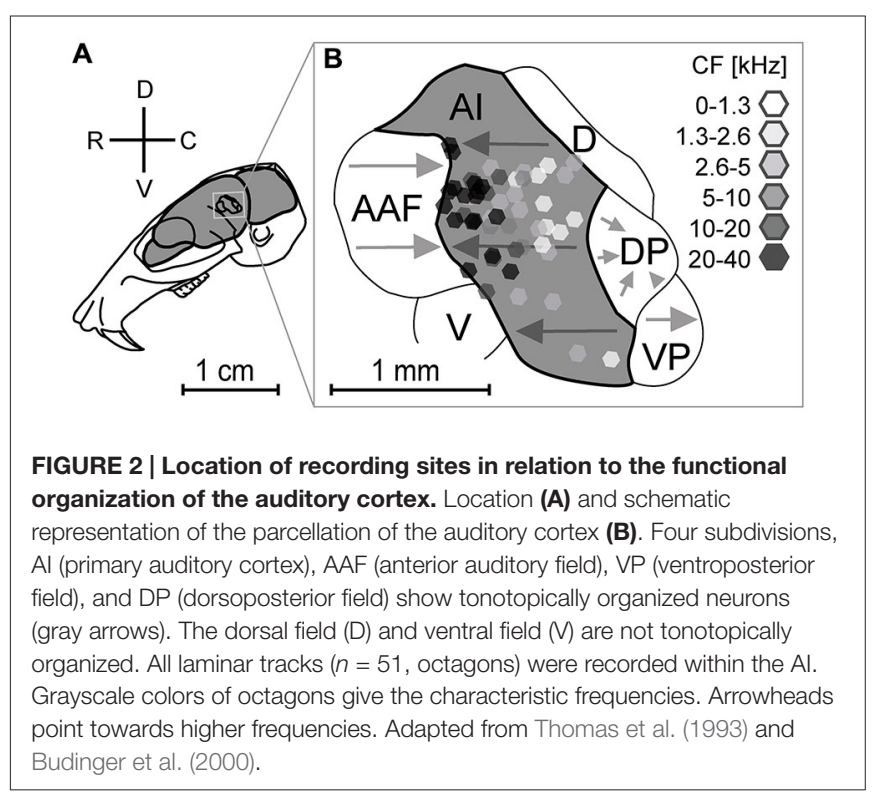


presenting a set of four stimuli differing in frequency and level. The neuronal signal was recorded in parallel from 16 equally spaced $(100 \mu \mathrm{m})$ channels from the pia mater to the white matter. Using the LFPs, one dimensional CSD patterns were calculated. The CSDs are essentially an ensemble activity mainly caused by excitatory synapses. The vertical extent in depth of each sink and its corresponding source (or sources) indicates the region over which the activated cells extend their dendrites (Mitzdorf, 1985). In the following, we characterize the current sinks with focus on the mid and late evoked components and quantify the changes in the CSD patterns to spectrally distant and suprathreshold stimuli, to assess their relative contributions to the responses' temporal structure.

\section{Frequency Representation of Neurons in the Al}

To investigate the current sinks that appeared in response to different stimuli, we first characterized the frequency tuning of each recording site by presenting pure tones of varying frequency and intensity. The neurons of the tonotopically organized core area AI are known to respond to narrow frequency ranges with short latencies of $\sim 10-25 \mathrm{~ms}$ (Thomas et al., 1993) and receive cochleotopic input directly from the ventral and medial division of the medial geniculate body (Wessinger et al., 2001; Linden and Schreiner, 2003). Due to a columnar cortex organization, the neuronal CF remains relatively similar in each layer (Wallace and Palmer, 2008) and therefore we considered it sufficient for the characterization of the entire penetration site to analyze the tuning curve shape and CF at one depth. Two representative tuning curves of different animals are displayed in Figures 3A,B. Tuning curves showed characteristic V-shapes with higher spiking activity at increasing sound pressure levels. The CF (red asterisks) at the peak of the tuning curves, which were interpolated at $30 \%$ of the maximum spiking rate, were calculated for each electrode penetration at layer V/VI. In this layer stimuli elicited the shortest onset latencies for sink s2 (Figure 1C). The frequency of the CF80, the $80 \mathrm{~dB}$ SPL pure tone at which the CSD patterns were analyzed, was adjusted in a way that it matched the CF calculated from the tuning curves calculated for layer V/VI.

In Figure 3C the CF is displayed against the MT of all tuning curves measured in layer V/VI. In gray, the global outline of all shapes of respective receptive fields is plotted. The shape of this threshold curve corresponds to the behavioral hearing threshold curve of Ryan (1976), but thresholds were about 5-15 dB less sensitive than in the behavioral data. The hearing-sensitivitydecrease between 5 and $15 \mathrm{kHz}$ was more pronounced in the present study and was probably due to the sparse distribution of neurons having their $\mathrm{CF}$ at this frequency range. The matching of the frequency of the four stimuli with the respective receptive fields is displayed in Figure 3D. As one would expect, in 100\% of the cases, the receptive fields of the studied neurons overlapped with the frequencies of CF80 and CF24+ stimuli. The frequency of -1 oct and +1 oct matched in 96 and $66 \%$ with the respective receptive field. In most cases (81\%), in which the frequency content of +1 loct appeared outside of the receptive fields, the CF was found to be above $20 \mathrm{kHz}$ which drove the frequency of +1 oct to the upper end of the animals hearing range.
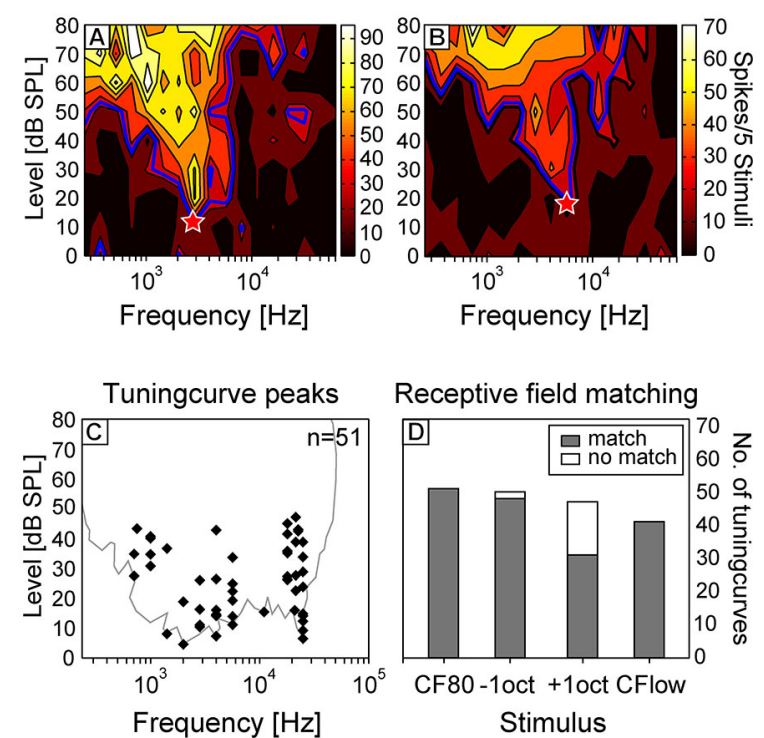

FIGURE 3 | Analysis of receptive fields of cortical neurons.

Representative tuning curves of two neurons of different animals are displayed in the upper row [characteristic frequency (CF; red asterisks) $=2.8 \mathrm{kHz}(\mathbf{A})$; $5.7 \mathrm{kHz}(\mathbf{B})$ ]. Tuning curves were interpolated at $30 \%$ of the maximum spike rate. (C) CF displayed against minimum threshold (MT) of all tuning curves measured at layer V/VI of each cortical site. In the background the outline of all shapes of respective receptive field is plotted. Only neurons with sensitive receptive fields (MT below $50 \mathrm{~dB}$ SPL) were selected for this study. (D) The percentage of receptive fields matching with the respective frequency content of the four stimuli.

\section{Changes of CSD Patterns to Different Stimuli}

In our study, we could identify two initial (s1 and s2), three mid (s3, s4, and s5), three late evoked sinks (s6, s7, and s8, see Figure 1C; colored in red), and one late sink (s9) appearing in a few cases within a $600 \mathrm{~ms}$ recording window. The initial sinks s1 and s2 characterized by the shortest latencies were located in the thalamic input layers III/IV and V/VI and from now on they will be referred to as primary sinks. Primary sinks appeared to be the most prominent and most reliably evoked components in the CSD patterns observed across the analyzed stimuli. The secondary sinks (s3-s8) were more variable and less reliably evoked across laminar profiles.

Two examples of CSD patterns of different animals obtained with different stimuli at two cortical sites are displayed in Figures $\mathbf{4 A - H}$. The corresponding tuning curves interpolated from spike data at $950 \mu \mathrm{m}$ depth (layer VI; CF $=2.8 \mathrm{kHz}$; $5.7 \mathrm{kHz}$ ) are displayed in Figures $\mathbf{3 A}, \mathbf{B}$. Sink contours were calculated at $8 \%$ of the maximum sink strength of the respective CSD pattern elicited by CF80 stimulation. The examples in Figures $\mathbf{4 A}-\mathbf{H}$ illustrate the variability observed in the CSD patterns. Although there were differences between the two sets of CSD patterns, they were qualitatively very similar and could be interpreted as reflecting the same basic pattern of excitatory synaptic activations. Over all recording sites, the earliest sink of CF80 stimulation (the one with the shortest latency) was s2 [11.7 $\pm 2.7 \mathrm{~ms}$ (mean $\pm \mathrm{SD}$ )] which appeared at a depth of $867 \pm 254$ $\mu \mathrm{m}$ (mean $\pm \mathrm{SD}$ ) followed by sink s1 [15.5 $\pm 4.6 \mathrm{~ms}$ (mean \pm 


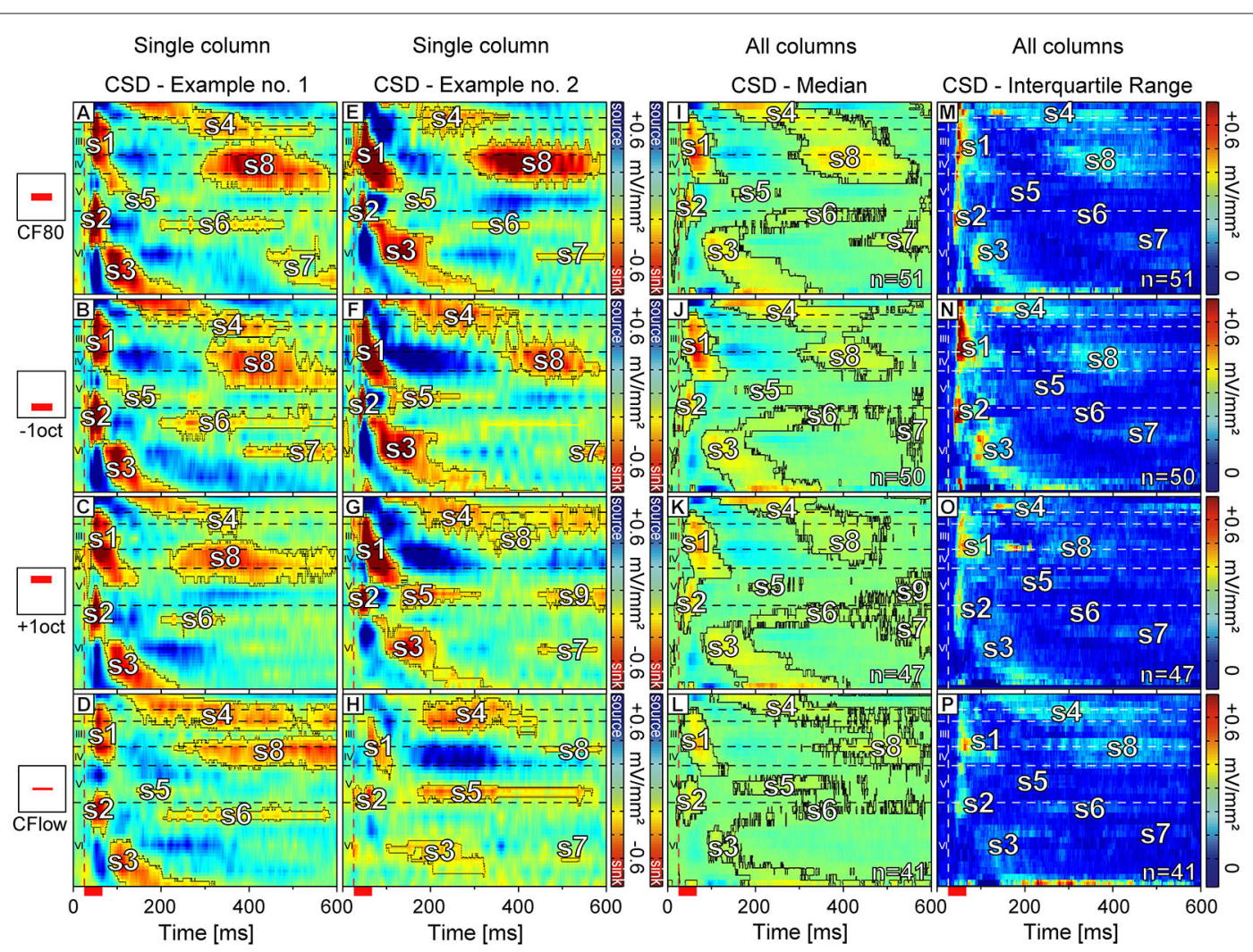

FIGURE 4 | Color maps of representative and averaged laminar CSD profiles. In the first and second column (A-H) representative CSD profiles of the same cortical column are shown. Neurons were stimulated with four different stimuli: sinus at CF at $80 \mathrm{~dB}$ SPL (A,E), sinus at CF one octave below (B,F), sinus at CF one octave above (C, G), and sinus at CF with a level set $\leq 24 \mathrm{~dB}$ above the individual MT (D,H). Sink contours were calculated at $8 \%$ of the maximum sink strength of the respective CSD pattern elicited by CF80 stimulation. (I-L) The median of laminar profiles averaged over all recording sites for each stimulus respectively. Sink contours were calculated at $4 \%$ of the maximum sink strength of the respective CSD pattern elicited by CF80 stimulation. (M-P) The interquartile range of sinks for each stimulus group specifically. Horizontal dashed lines indicate layer borders while the vertical dashed line marks the beginning of pure tone stimulation. Red scale bar represents stimulus duration of $30 \mathrm{~ms}$.

SD)] with a small delay $[4 \pm 3.9 \mathrm{~ms}$ (mean \pm SD) $]$ at a depth of $320 \pm 119 \mu \mathrm{m}$ in layer IV. Secondary sinks emerged in layer V (s5), layer VI (s3), and layer I (s4). The sink s6 followed with a short delay in layer VIa. Latest sinks arose in thalamic input layers III and IV (s8) and layer VI (s7). Some primary sinks of cortical sites [s1 (23\%); s2 (47\%)] showed pre-discharges which were artifacts produced by the digital notch-filter and were thus excluded.

CSD patterns emerging from +1oct (Figures $4 \mathbf{C , G}$ ) and CF24+ (Figures $4 \mathbf{D}, \mathbf{H})$ had in comparison to patterns emerging from CF80 (Figures 4A,E) and -1oct (Figures 4B,F) lower strength for s1-s4 and s8. The strength of s5-s7 remained relatively low independent of the applied stimulus. The patterns evoked by CF80 and - 1oct shared similarities concerning the sink shape and duration for s1-s6. Patterns elicited by CF24+ stimulation showed a rather different structure, when compared to all other stimuli (Figures 4D,H). Primary sinks had longer onset latencies [s1 delay: $24.29 \pm 11.3 \mathrm{~ms}$; s2 delay: $19.3 \pm$ $11.6 \mathrm{~ms}$ (mean $\pm \mathrm{SD}$ )] and secondary sinks s4, s5, and s6 -if present- were prolonged in duration in comparison to responses to CF80 [s1 delay: $15.5 \pm 4.6 \mathrm{~ms}$; s2 delay: 11.7 $\pm 2.7 \mathrm{~ms}$ (mean $\pm \mathrm{SD}$ )]. In the CSD profile arising from +1oct stimulation (Figure 4G) a new sink s9 appeared that was located in layer V. This sink was spatially separated from s5 and there was no clear trend that s9 could be associated with specific CFs or MTs. In some profiles (see Figures $4 \mathbf{G}, \mathbf{H}$ ) +1 oct and CF24+ stimulation evoked s8 sinks (defined as s8 only if clearly separated from s4) with their point of maximum activity shifted towards layer III. Remarkably, s7 was often missing or the sink strength did not surpass the applied criterion (Figures 4C,D). The overall variability regarding the presence and the area size was increased especially for the secondary sinks.

The exemplary CSD patterns shown in Figures $\mathbf{4 A - H}$ reflect the data at the population level (Figures 4I-L). All recordings were aligned in depth and merged together for each stimulus respectively. Each point in the pattern represents the median value. In contrast to the individual examples of CSD patterns (Figures $4 \mathbf{A}-\mathbf{H}$ ) the sink contours were calculated at $4 \%$ of the maximum sink strength of the median CSD pattern evoked by CF80 stimulation (Figure 4I). This criterion had to be applied as the median calculation lead to a reduced sink and source strength due to the relatively high variance of CSD patterns. Sink s7 was faintly present (Figures 4J-L), likely due to its unreliable nature 
in individual laminar profiles. For +1 loct stimuli (Figure $4 \mathrm{~K}$ ), sink s9 appeared also at the population level with a relatively strong maximum strength in layer $\mathrm{V}$.

As mentioned before, CSD patterns even within the same stimulus group were relatively variable. To account for these variances and to quantify the extent and location of the different sinks, we calculated the interquartile range exclusively for negative values (sinks) of each stimulus group specifically (Figures 4M-P). The highest variances could be observed for sinks s1, s2, and s3 in the - 1oct patterns. These are also known to be the most prominent sinks regarding their strength and also showed relatively high variances for the other stimuli. While the 55 variances evoked by CF80 remained low, they were more pronounced for -1 oct and +1oct. Sink s6 showed the highest variances during CF24+ stimulation. The variances for s4, s7, and s8 were relatively small for all stimulus groups.

\section{Reliability of Evoked Sinks}

As the variances between laminar profiles are dependent on the occurrence of sinks, the percentage of sinks for the different stimulations is displayed in Figure 5. The most reliably evoked sinks were primary sinks s1 and s2 and secondary sinks s3, s4, and $s 8$ (mean $=96 \%)$. Sinks s5 and s6 were less reliably evoked and appeared in $86 \%$ of profiles. Sink s7 at CF80, - 1oct and +oct was present at the same level as $s 5$ and s6 but only present in $39 \%$ for CF24+ stimuli. The laminar depth of the maximum activity of s8 (s8s) elicited by +1oct and CF24+ was located in 62 and $49 \%$ of laminar profiles in layer III, respectively. This shift was present in $29 \%$ in CF80 and in $34 \%$ in -1oct. The sink s9, which was rarely present for $-10 c t, \mathrm{CF} 80$, and CF24+ $($ mean $=$ $21 \%$ ), was strongly present in $68 \%$ of profiles for +1 oct stimuli. Regarding s1-s8, CF80 stimulation led to the most stable evoked sinks with an average occurrence-rate of $95 \%$, followed by -1 oct (94\%), +1oct (92\%) and CF24+ (82\%).

\section{Quantitative Analysis of Sink Parameters}

To quantify the differences of the stimulus-specific CSD profiles we plotted contours around the sinks of laminar profiles, which surpassed $8 \%$ of the maximum sink amplitude elicited by CF80

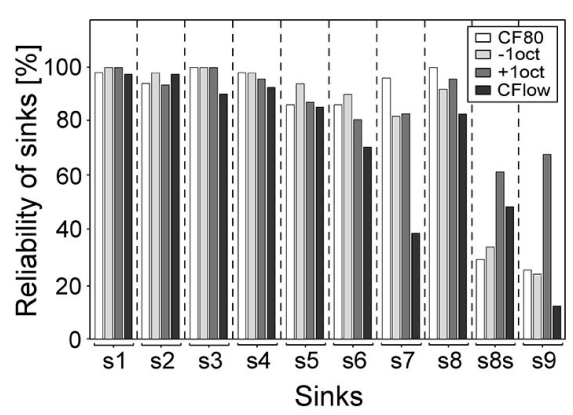

FIGURE 5 | Reliability of sinks. Bars give the percentage of presence of sinks for the different stimuli. Sinks s1-s4 and s8 were present in most of the laminar profiles (mean $=96 \%$ ). Sink s7 of CF24+ was absent in $61 \%$ of profiles. The maximum activity of $\mathrm{s} 8$ (s8s) elicited by +1 oct and CF24+ was located in 62 and $49 \%$ of laminar profiles above the layer III/IV border. The sink s9 of +1 oct was present in $68 \%$ of the laminar profiles. and calculated the maximum strength, onset latency, duration and vertical extent of each sink. In the following, we focus on s1, s3, s5, and s8 and present the changes between CF80, - 1oct, +1oct, and CF24+ stimulation. We chose the aforementioned sinks because they represent the primary thalamocortical input (sink s1), and the strongest secondary sinks of granular layer IV (s8), infragranular layer V (s5) and VI (s3). We assumed that analyzing those sinks could be enough to quantitatively access the stimulus-specific characteristics of sinks and layer dependent differences. A more detailed analysis of the differences between all sinks can be found in the supplementary information (Supplementary Figure 1).

We found low level pure tones $(\mathrm{CF} 24+)$ and pure tone frequencies of one octave distant from CF $(-1$ loct and +1oct) to evoke significantly different secondary sinks compared to CF80, indicating that different stimuli activate different cortical circuits. The maximum sink strength (Figure 6A) showed a relatively comparable pattern for s1, s3 and s8, in which CF80 and -1oct mostly elicited significantly higher maximum sink strengths when compared to +1 oct and CF24+. Similar maximum sink strengths for $\mathrm{s} 1\left(-1.11 \mathrm{mV} / \mathrm{mm}^{2}\right)$ could be evoked with best frequency stimuli by Happel et al. (2010). Sink s5 showed a completely different behavior with nonsignificant differences and relatively comparable values across analyzed stimuli. This is interesting, as it could indicate that s5 is generated by a mechanism different than that responsible for $\mathrm{s} 1$.

The onset latency (Figure 6B) is a fundamental descriptor of neuronal responses and has been studied in several works (Phillips and Irvine, 1981; Phillips et al., 1985; Foeller et al., 2001; Hagemann et al., 2010; Hechavarria et al., 2013). For the primary sink s1 and the secondary sink s3, we found that significantly longer onset latencies could be elicited by low level stimulation (median $=21 \mathrm{~ms}, 64.6 \mathrm{~ms}$ ) in comparison to CF80 (median $=14.6 \mathrm{~ms}, 50.8 \mathrm{~ms}$ ). That latency decreases with stimulus amplitude is a common feature, which is already present at the level of the auditory nerve as described in several studies (Picton et al., 1974; Phillips and Irvine, 1981; Polich et al., 1988; Mendelson et al., 1997). The two stimuli spectrally distant from the CF $(-1$ oct and +1 oct $)$ did not follow the same trend, although sharing the same octave distance. While the s1 and $s 3$ obtained at -1 oct stimulation were significantly different to s1 and s3 obtained during CF24+ stimulation, the median of s1 and s3 showed a non-significant latency shift of 3.6 and 8.4 ms during +1oct stimulation, which corresponds to previous studies (Kaur et al., 2005; Happel et al., 2010). The latencies of s5 and s8 during CF24+ stimulation had similar values in comparison to CF80, - 1oct, and +1oct stimuli, although the variance was high. In all four groups, sink s1 [likely elicited by inputs from the ventral division of the geniculate body (MGv)] showed longer latencies [median s1-s2: $3.2 \mathrm{~ms}$ (CF80); $3.2 \mathrm{~ms}$ (-1oct); $6.5 \mathrm{~ms}$ (+1oct); $3.7 \mathrm{~ms}(\mathrm{CF} 24+)]$ in comparison to s2 (see Supplementary Figure 1B), which is thought to be created by the medial division of the geniculate body (MGm; Linden and Schreiner, 2003).

The duration of primary sink s1 (Figure 1C) showed no significant differences between the four stimulus groups, but 

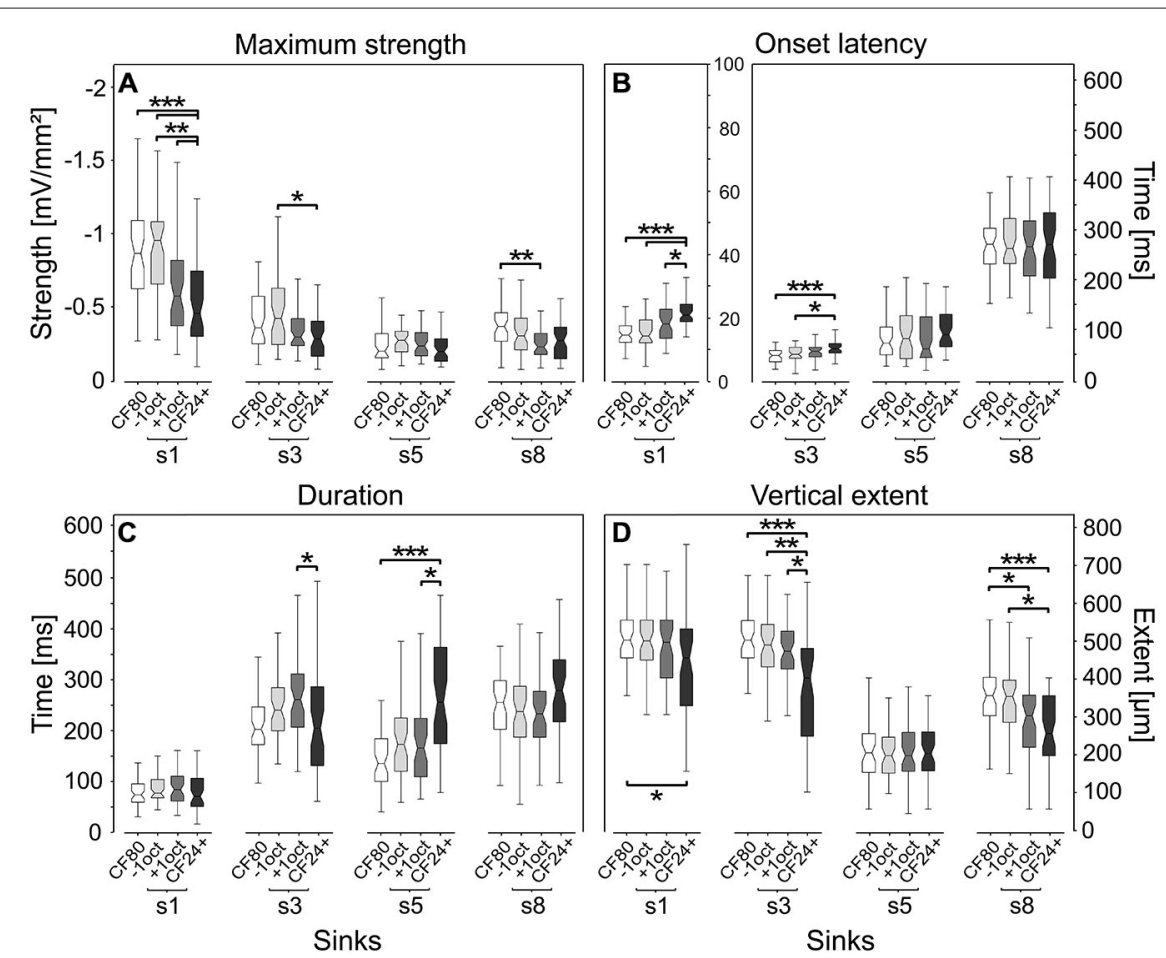

FIGURE 6 | Quantitative comparison of sink parameters. Parameters were calculated at $8 \%$ of the maximum sink strength of the respective CSD pattern elicited by CF80 stimulation. Low level pure tones (CF24+) and pure tone frequencies of one octave distant from CF ( -1 oct and +10ct) evoke significantly different sinks. Primary sink s1 (see sinks schematic above) and the most strongly elicited secondary sinks from infragranular layer VI (s3) and V (s5) and granular layer IV (s8) are compared. Four parameters were quantitatively assessed: (A) maximum sink strength, related to the strength of neuronal activity. (B) Sink onset latency, note the different y-axes for s1 vs. other sinks. (C) Sink duration. (D) Sink vertical extent. Boxplot whiskers represent data range, outer edge of box represents second and fourth quartiles of data, and midline represents median of data. Significance was determined using Kruskal-Wallis one-way ANOVA in combination with a multiple comparison post hoc test: ${ }^{*} p<0.05,{ }^{* *} p<0.01,{ }^{* * *} p<0.001$.

was longer than the duration of the phasic-like activation of neuronal spikes which were about $19 \mathrm{~ms}$ for CF80, -1oct, and +1oct stimuli and about $14 \mathrm{~ms}$ for CF24+ stimuli (measured as the duration of suprathreshold spikes at 3.5 times the standard deviation beneath the baseline). Differences at the level of significance $(p<0.05)$ were observed for $s 3$ and s5, both with opposite trends. While the duration of s3 was significantly shorter for CF24+ (median $=202 \mathrm{~ms}$ ) than for +1 oct (median $=258 \mathrm{~ms}$ ), the duration of s5 was significantly longer for CF24+ (median $=253 \mathrm{~ms})$ than for CF80 $($ median $=133 \mathrm{~ms})$ and +1 oct $($ median $=163 \mathrm{~ms})$. The same trend could be observed for $s 8$ but the differences were not significant.

The vertical extent (Figure 6D) revealed a homogeneous pattern for primary sink s1 and secondary sink s3 and s8, in which low level elicited sinks (median $=457 \mu \mathrm{m}, 404$ $\mu \mathrm{m}, 258 \mu \mathrm{m})$ had the significantly shortest vertical extents in comparison to CF80 (median $=504 \mu \mathrm{m}, 504 \mu \mathrm{m}, 357 \mu \mathrm{m}$ ). While s1, s3, and $\mathrm{s} 8$ of +1 oct $($ median $=498 \mu \mathrm{m}, 474 \mu \mathrm{m}$, $304 \mu \mathrm{m}$ ) followed the same trend, it was only at the level of significance for s8. Interestingly, the vertical extent of sink s5 appeared to be unaffected by different stimuli and showed no significant differences similar to the maximum strength or onset latency.

\section{Correlation of Laminar CSD Profiles}

Significant stimulus-specific differences in the CSD patterns could be found at the level of sink parameters (see Figure 6). To be able to account for the overall difference of laminar patterns, including sinks, sources and background noise, we also calculated correlation coefficients between CSD patterns obtained for all different stimuli compared to the control CF80. The latter was done for each recording tract separately. In Figure 7 the results are displayed as boxplots. The correlation between CF80 and -1 oct or +1 oct was significantly higher $(r=0.69 \pm 0.3$; $r=0.53 \pm 0.3)$ than the correlation between CF80 and CF24+ $(r=0.38 \pm 0.25)$. Most significant differences in the sinks (see Figure 6) could be observed for the low level CF24+ stimulus, which also yields the weakest correlation with the response obtained with CF80 stimuli.

\section{Discussion}

\section{Methodological Considerations and Limitations}

LFP are influenced by nonspecific factors such as ongoing cortical activity (spontaneous or evoked by a preceding stimulus) or the state of excitability (Mitzdorf, 1987). Most anesthetics appear to decrease neural conduction and synaptic transmission, thereby decreasing the amplitude and increasing the latency of 


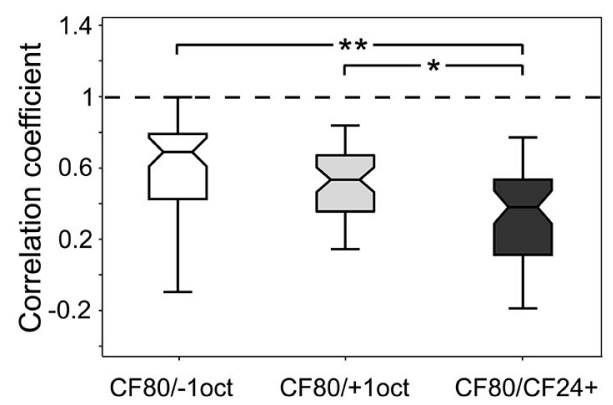

FIGURE 7 | Stimulus-specific correlation of laminar CSD profiles. Boxplots show the correlation coefficient between the laminar profiles of CF80 compared to the remaining stimuli ( $-10 c t,+10 c t$, and $\mathrm{CF} 24+)$. Boxplot whiskers represent data range, outer edge of box represents second and fourth quartiles of data, and midline represents median of data. Significance was determined using Kruskal-Wallis one-way ANOVA in combination with a multiple comparison post hoc test: ${ }^{*} p<0.05,{ }^{* *} p<0.01$.

evoked responses (Armstrong-James and George, 1988; Kuwada et al., 1989; Zurita et al., 1994; Sloan, 1998; Gaese and Ostwald, 2001). Considerable contribution to variances of CSD patterns could come from up and down states, which are associated with different states of cellular excitability that effectively influences evoked potentials by generating either increased or decreased amplitudes (Timofeev and Steriade, 1998; Petersen et al., 2003; Saleem et al., 2010). Such effects may be stronger for mid (Mitzdorf, 1987) and long latency sinks as many sources could contribute to their generation.

The CSD technique provides insights into the activation patterns of ensembles of cortical neuronal populations at sublaminar dimension, but this method employs a number of assumptions, such as isotropic current in the tangential direction and uniform conductivity, neither of which is strictly accurate for the cerebral cortex. Aside from this, Tenke et al. (1993) pointed out that the accuracy with which the CSD analysis can be related to the activation of underlying neural elements is influenced by noise and spatial resolution. The use of uniformly manufactured commercially available multicontact electrodes utilized in the present study allowed us to minimize interchannel distance inaccuracies. The temporal sampling resolution is crucial for adequately representing activity alterations having a high frequency, whereas the spatial sampling resolution is important for gathering detailed information on subpopulations of participating neurons. In the present study, the temporal resolution was much higher $(20 \mathrm{kHz})$ than that required for LFP measurements whose frequencies are defined to be below $300 \mathrm{~Hz}$. The spatial resolution (channel spacing of $100 \mu \mathrm{m}$ ), which proved useful in previous studies in rats (Kaur et al., 2005; Szymanski et al., 2009), was supposed to be adequate to identify functional interactions that proceed within individual cortical laminae or sublaminae, but could also magnify the contributions of computational artifacts (Tenke et al., 1993). However, CSD patterns are superpositions of multiple corresponding sinks and sources. At longer latencies, a larger number of sources and sinks can interfere, which makes it increasingly difficult to separate them. Therefore late sinks do not necessarily reflect a "true sink component" in the sense of a mass trans-membrane current at a certain location and latency and should be considered critically.

\section{The General Structure of CSD Flow-Patterns in the Mammalian Neocortex}

The more or less uniform neuronal architecture of the neocortex and its six-layered structure corroborate the hypothesis that afferent activity is relayed very similarly in all sensory areas and reflects the same types of excitatory synaptic ensemble activities along the same intracortical pathways thus leading to similar CSD patterns (Atencio and Schreiner, 2010). Several studies in the visual cortex of monkeys (Mitzdorf and Singer, 1979; Kraut et al., 1985), cats (Mitzdorf, 1985, 1987), and rabbits (Rappelsberger et al., 1981; Pockberger and Rappelsberger, 1983), in the somatosensory cortex of rats (Di et al., 1990) or in the auditory cortex of monkeys (Müller-Preuss and Mitzdorf, 1984; Steinschneider et al., 1992), rats (Kaur et al., 2005; Szymanski et al., 2009) or gerbils (Happel et al., 2010) led to similar patterns where early sinks (s1 and s2) were present in the input layer IV [and deep layer III in the somatosensory or auditory cortex (Huang and Winer, 2000; Lee and Winer, 2008)] and in many studies [mostly in the auditory cortex (Mitzdorf, 1985)] at the layer V/VI border. Later mid latency sinks in layer II, $\mathrm{V}$, and VI were apparent in most of these profiles with a considerable delay and strongly resemble s4, s5, and s3 of the present study (Pockberger and Rappelsberger, 1983; Mitzdorf, 1985, 1987; Di et al., 1990; Lakatos et al., 2007; Happel et al., 2010; Kajikawa and Schroeder, 2011). However, qualitatively different CSD patterns were found in the visual (Lakatos et al., 2009) and auditory cortex (Lakatos et al., 2007, 2009; Kajikawa and Schroeder, 2011; O’Connell et al., 2011; Tenke and Kayser, 2012) of monkeys, where the activation sequence in layers II-IV was intermediated by a source in layer III. Architectonical differences in the laminar structure of the monkey cortex, indicated by differently proportionate layers and concurrent broadening of supragranular and compression of infragranular layers (Lakatos et al., 2005; Maier et al., 2011; O'Connell et al., 2011) could serve as an explanation. There are no other studies on late sinks s6-s9 available for the auditory cortex. In the visual cortex of cats late sinks were present in layers III, V, and VI, which could resemble s8, s6, and s7 of the present study, but showed far longer onset latencies (Mitzdorf, 1985, 1987).

\section{Neuronal Origins of Early Evoked Sinks}

In the present study, s1, located in the input layers III and VI, likely reflects lemniscal thalamocortical input from tonotopically organized afferent inputs from the ventral part of the medial geniculate body (MGv; Budinger et al., 2000; Huang and Winer, 2000). Small pyramidal cells in layers IIIb and IV seem to be the main thalamorecipient neurons in auditory cortex (Linden and Schreiner, 2003; Wallace and Palmer, 2008). Experiments on silencing cortex by the $\mathrm{GABA}_{\mathrm{A}}$ agonist muscimol further confirmed the contribution of cortical neurons on the generation of s1 (Kaur et al., 2004; Happel et al., 2010). Sink s2, located at the layer V/VI border, was present in almost every CSD pattern (see Figure 5) and likely reflects non-lemniscal thalamocortical 
input from the medial part of the medial geniculate body (MGm; Steinschneider et al., 1998; Kimura et al., 2003; Linden and Schreiner, 2003; Winer and Lee, 2007). These deep initial sinks had shorter onset latencies [11.4 ms (see Supplementary Figure 1)] than the sinks in infragranular layers III-IV (14.6 ms), which was in accordance with previous studies in the primary auditory cortex of rodents [rat (Kaur et al., 2005; Szymanski et al., 2009), gerbil (Sugimoto et al., 1997), mouse (Shen et al., 1999), and guinea pig (Wallace and Palmer, 2008)].

\section{Neuronal Origins of Mid and Late Evoked Sinks}

To our knowledge, no quantitative study thus far has characterized the late sinks (s6-s9). While the origins of the early initial sinks are known to a large degree, those of the successive mid and late cortical activations are rather obscure and proposed to be composed of an ensemble activity generated by different areas from the primary and secondary cortical fields, the FC, the centro-parietal cortex, association cortex, and/or the hippocampus (Picton et al., 1974; Skrebitsky and Sharonova, 1976; Kraus et al., 1985; Mitzdorf, 1985; Näätänen and Picton, 1987; Barth and Di, 1990; Santos Filha and Matas, 2010). With our current data we cannot resolve the neuronal origins of mid and late evoked sinks. Although mid and late components might have an important role in the cortical processing, as they are more or less reliably evoked. It is difficult to speculate about the sources of these sinks, as the temporal gap between initial excitatory and delayed activity is approximately $50 \mathrm{~ms}$ (comparing the onset latencies). This represents a relatively long cortical processing time during which many possible mechanisms could operate. Di et al. (1990) proposed that the later components in the CSD patterns of the rat barrel cortex represent hyperpolarizations or repolarizations. However, several other studies assumed different mechanisms for mid and late sinks assignable to at least three categories of synaptic input: (1) repetitive after-discharges from thalamus relay neurons; (2) intrinsic processing within the AC microcircuits; and (3) inputs from outside of the AC such as the FC, the contralateral hemisphere or the hippocampus. We will discuss these possibilities in the same order as depicted above.

(1) Repetitive thalamocortical after-discharges as a generator of mid and late sinks has been described in the visually cortex of rabbits (Rappelsberger et al., 1981) and cats (Mitzdorf, 1985). They occurred rather consistently when the animal was deeply anesthetized and/or its neurons exhibited low excitability. Mitzdorf (1985) observed that these after-discharges usually induce only small sinks in the input layers and usually recurred a few times with successively lower amplitudes and longer intervals. It has been suggested that these after-discharges are generated in the thalamus (Buser and Horvath, 1972; Horvath and Buser, 1972) or in the retina in the case of the visual cortex (Wachtmeister and Dowling, 1978) and are conducted to the cortex via specific afferents. Taken the thalamocortical wiring into account, in which MGv projects to layers III, IV, and VIb and MGm to layers I, Vb, and VIa, most of the sinks (s3, s4, s6, s7, and s8) could be explained by comparing the thalamocortical target layers with the cortical depth of the earliest activity within a sinks, in short onset latency depth (Figure 8). As pyramidal neurons in layers $\mathrm{Va}$ and $\mathrm{VIb}$ are the main sources of thalamic

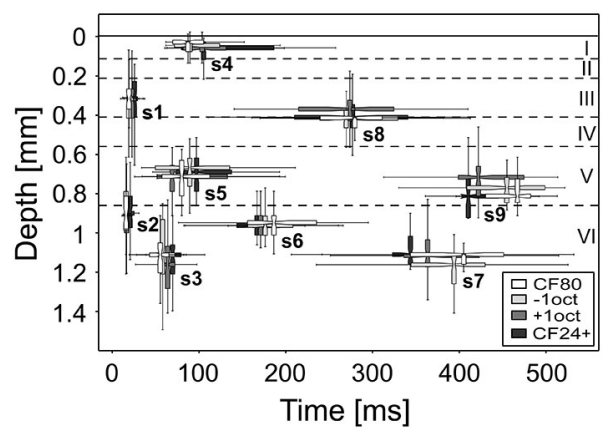

FIGURE 8 | Onset latency and depth of stimulus-specific sinks. The range of the onset depth is displayed at the median of the respective onset latency. The sinks remain relatively constant regarding the layer and time at which the neuronal activity is evoked. Boxplot whiskers represent data range, outer edge of box represents second and fourth quartiles of data, and midline represents median of data.

feed-back projections (Linden and Schreiner, 2003), s5 and s7 both having similar maximum strength could originate from reciprocal inputs (Figure 9). However, pyramidal neurons are not exclusively activated by thalamocortical inputs as they form connections to many different ipsi- and contralaterally located non-thalamic neurons (Mitani et al., 1985; Linden and Schreiner, 2003; Thomson and Lamy, 2007; Izhikevich and Edelman, 2008).

(2) The contribution of intrinsic cortical connections could also serve as an explanation for mid and late evoked sinks. Kaur et al. (2004) could show a partial suppression of initial and a full suppression of longer-latency response components with muscimol, suggesting a major involvement of intracortical pathways. However, it is not clear to what extent activity from the primary auditory cortex contributes, directly or indirectly to mid or late evoked sinks. It is known that the majority of synapses in a cortical column are intrinsically connected and less innervated by thalamic or distant cortical areas (Douglas and Martin, 2007). Five to twenty percent of the inputs to the granular layer IV originate from convergent thalamocortical and $80-95 \%$ from intracortical projections (Peters et al., 1994; Ahmed et al., 1997; Logothetis, 2008). It is known that intrinsic vertical and horizontal connections (Figure 9) influence the cortical network (Mitani et al., 1985; Matsubara and Phillips, 1988; Read et al., 2001; Atencio and Schreiner, 2010) and could thus dominate the generation of later sinks. But it is still unclear if the intracortical synapses are related to local intracolumnar or intercolumnar neurons, as it has been proposed based on anatomical data (Ojima et al., 1991, 1992; Budinger et al., 2000). In addition, there are parallel excitatory and inhibitory circuits (feed-forward and feed-back) in which different inhibitory interneurons play an important role in the regulation of cortical operations (Shepherd and Koch, 1998; Kanwal et al., 1999) that could further contribute to the shaping of late sinks. However, the contribution of inhibitory postsynaptic potentials to the sinks is at least one order of magnitude smaller in amplitude than the contribution of excitatory postsynaptic potentials (Mitzdorf, 1987; Schroeder et al., 1990; Tenke et al., 1993). 


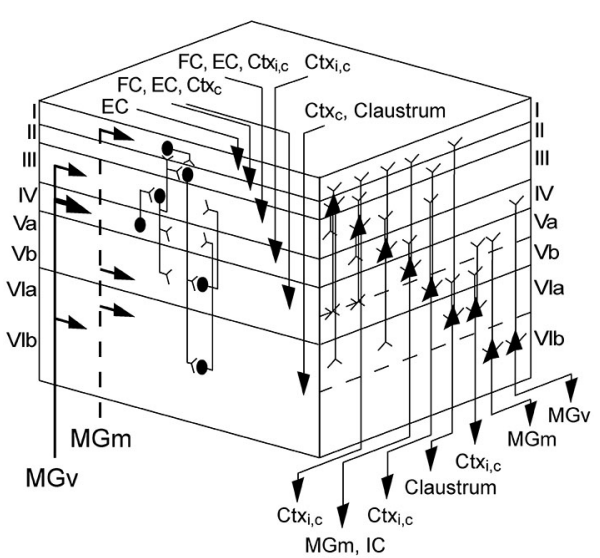

FIGURE 9 | Cortical and laminar network organization of the AC. Left face of cube shows inputs from the ventral (MGv) and medial divisions (MGm) of the geniculate body and cortical areas and a schematic of the interlaminar connections; right face displays the pyramidal neurons of each layer and gives their axonal targets. Note that this schematic drawing illustrates only the major pyramidal neurons. Lemniscal thalamic inputs from MGv mainly arrive at layers III and IV, but also at layer VI. Nonlemniscal inputs from MGm activate layers I, $\mathrm{Vb}$, and $\mathrm{Vla}$. Thalamic activation of layer IV initiates a flow of information into the supragranular layers I-III and then down to the infragranular layers $V$ and VI. Corticocortical inputs from the ipsilateral hemisphere $\left(\mathrm{Ctx}_{\mathrm{i}}\right)$ terminate in the middle layers III and IV. Commissural inputs $\left(\mathrm{Ctx}_{\mathrm{C}}\right)$ are widely distributed and arrive in almost all layers (II-VI). Inputs from the claustrum terminate in layer $\mathrm{VI}$, whereas the entorhinal cortex $(\mathrm{EC})$ and frontal cortex $(\mathrm{FC})$ projects to neurons in layers (I) II, III, and V. Pyramidal cells are present in all layers, but layer I. The pyramidal axons of layers II-VI extend into layer I, with the exception of different pyramidal cell types in layers Vla and Vlb. Corticocortical projections emerge from layers III, V, and VI. Feed-back to the auditory thalamus originates primarily in layer $\mathrm{VI}$, but also in layer $\mathrm{V}$, which also projects to the inferior colliculus (IC). Adapted from Mitani et al. (1985), Insausti et al. (1997), Linden and Schreiner (2003), Mitchell and Macklis (2005), Thomson and Lamy (2007) and Izhikevich and Edelman (2008).

(3) Ferreyra-Moyano et al. (1988) demonstrated for the rat olfactory cortex that activation of pyramidal neurons from outside of the AC may be the main generators of late evoked components, rather than re-excitation of mitral cell axons. Certain types of secondary responses associated with complex brain functions are influenced by barbiturate resulting in either reduced or abolished activity (Fuster and Docter, 1962; Torres and Warner, 1962). These responses are correlated with activation of the mesencephalic reticular formation (Fuster and Docter, 1962; Torres and Warner, 1962) and are related to memory (John, 1967) and passive or active conditioning (Sakhiulina and Merzhanova, 1966; Fox, 1970). It is also believed that the FC, the centro-parietal cortex, and the hippocampus are possible generators for late components (Santos Filha and Matas, 2010). Evidence for an important role of mid latency sinks in somatic sensory perception has been suggested by a previous work which showed that depolarizations predicted the sensory discrimination performance of monkeys (Kulics and Cauller, 1986). In addition, using auditory and visual oddball tasks, Halgren et al. (1998) reported that sensory-specific areas have a long period of activity to which widespread brain circuits contribute at approximately the time of late sinks onset (s6-s9). The above mentioned circuits are related to the paralimbic and attentional frontoparietocingular cortex and to the eventencoding cortices, the association cortex, and the hippocampus. Neurons from the hippocampus project via the entorhinal cortex (EC) mainly to neurons in superficial layers I, II, and III and infragranular layer V of sensory cortices (Kosel et al., 1982; Insausti et al., 1997). As hippocampus and EC are related to spatial memory, navigation, and episodic memory (Jacobs et al., 2010), it is likely to assume, that repetitive stimulation can trigger neuronal activity in both areas contributing to the generation of late evoked sinks s8 and s9. Similar to the EC, the FC also targets layers II, III, and V (Mitchell and Macklis, 2005). It has long been suspected to play an important role in cognitive control, in the ability to determine actions in accordance with internal goals (Fuster, 2000; Kanwal et al., 2000; Sakamoto et al., 2015). Research based on surface recordings indicated that longlatency auditory evoked potentials probably arise from frontal associative areas (Picton et al., 1974; Iwasa and Potsic, 1982). As sink s8 showed a rather strong activation compared to most of the later sinks ( 55, s6, and s7), it could be evoked by a convergent interaction of several different brain areas such as the hippocampus or the FC. However, its reliability could indicate a stable source of generation e.g., thalamic feed-back projections to layer IV which would imply a more or less constant time delay between both sinks s1 and s8 (s5). But this is not the case as the time delay shortens in dependence of applied frequency or level (Figure 8) due to a latency shift in s1. Late sink s7 located in layer VIb could be evoked by inputs from contralateral cortices and/or the claustrum as suggested by cortical wiring (Linden and Schreiner, 2003; Thomson and Lamy, 2007). The fact, that s3, s4, s6, and s8 have a considerably longer duration than s5, s7 or s9 (see Supplementary Figure 1) could mean, that they reflect modulatory output.

In summary, we can propose a hypothetical scheme of the specific sink generators based on results of previous studies and on the cortical architecture. The early part of s1 and s2 is clearly of thalamic origin and the later part with a high possibility of intrinsic cortical origin. The mid latency sinks s3-s5 are most likely evoked by inputs from cortical areas due to the long latency. The late sinks s6-s9 are supposedly evoked by specific brain areas such as the frontal or EC, which are temporally correlated with these sinks (see Figure 8) and are associated with memory, learning or cognitive control.

\section{Change of Current-Flow-Patterns Due to Level and Spectral Integration}

We investigated differences in the CSD patterns, especially for the mid and late sinks, in relation to three different stimuli residing at the frequency and sensitivity corners of respective neuronal receptive fields. Our results show that the intracortical information-flow-patterns do not exhibit qualitative changes in response to the stimuli tested. Several studies proposed that short-range or long-range intracortical ("horizontal") connections could provide spectral input (excitatory and inhibitory) to differently tuned columnar neurons receiving spectrally different direct thalamic input (Kaur et al., 2004, 2005; Tomioka et al., 2005; Kurt et al., 2008; Happel et al., 2010; Moeller et al., 2010). Other studies demonstrate large scale spectral 
integration based on the receptive fields of single neurons (Kanwal et al., 1999) and assume that spectral information within the range of $\mathrm{CF} \pm 1$ octave is fed through direct thalamocortical projections to layer III/IV (Kaur et al., 2005; Happel et al., 2010; Guo et al., 2013). It has been shown that the laminar organization of horizontal and thalamocortical inputs are different in a way that stimulation near the frequency eliciting the highest firing rate led to activations of layer IIIa (Happel et al., 2010). This is in accordance with the excitatory horizontal inputs mainly terminating in layer II/III (Dantzker and Callaway, 2000; Thomson and Bannister, 2003). We could not observe this shift of activity in our dataset, as the cortical depth of the maximum strength within s1 was constantly located in layer III and the vertical extent remained similar for CF80 and near CF stimuli (see Supplementary Figures 1D,F). In regard to s8 located in layer III/IV we could observe a quite reliable shift in the depth of the maximum strength towards layer III for +1 oct and CF24+ stimuli (Figure 5) indicating a contribution of horizontal inputs. Most of the initial, mid, and late sinks showed partly significant differences such as maximum strength, onset latency, duration, and vertical extent already at a spectral distance of CF \pm 1 octave (Figures 6A-D). Stimulation with + loct produced a characteristic laminar activation profile in the initial granular sink s1 with (non-significantly) decreased maximum strength and an increased onset latency (and duration; Figures 6A-C), which was in accordance with previous works (Kaur et al., 2005; Happel et al., 2010; O'Connell et al., 2011). In comparison to CF80 stimulation the onset latency of s1 and s3 during +1 oct stimulation increases in contrast to s5 or s8 (Figure 6B), supposing s3 as a direct or indirect (via s1 and s2) target for spectral integration fibers initializing a feed-forward activation cascade (Happel et al., 2010; Guo et al., 2013). In contrast to this, the same spectral distance from the respective CF in the opposing direction (-1oct) produced initial sinks (and to a certain degree also mid and late sinks) which resemble the ones of CF80 stimulation in terms of maximum strength, onset latency and vertical extent (Figures 6A,B,D, 8). This could indicate that both +1 oct and CF24+ follow comparable processing mechanisms or at least activate similar neuronal clusters. Discrepancies in the onset latencies evoked by spectrally different stimuli could also be observed by Guo et al. (2013). In contrast to our data, in the Guo study both spectrally distant CF \pm 1 octave stimuli elicited longer s1 onset latencies of which +1 oct $(\sim+5 \mathrm{~ms})$ is similar to our data $(+3.6 \mathrm{~ms})$ but not the -1 loct $(\sim+6.5 \mathrm{~ms}$ vs. $-0.2 \mathrm{~ms}$ ). However, both results are hard to compare as different sound levels ( $80 \mathrm{~dB}$ SPL vs. $\sim 30-35 \mathrm{~dB}$ SPL) were used, but this could indicate a level dependent mechanism of spectral integration.

When comparing the whole CSD pattern, the tendency of -1 oct eliciting similar sink parameter as CF80 is reflected by a higher correlation coefficient $(r=0.69 \pm 0.3)$ than for CF80 and +1 oct $(r=0.53 \pm 0.3$, Figure 7$)$. One reason for a discrepancy between two equally spectrally distant stimuli could originate from asymmetrically shaped tuning curves. This shifts +1oct stimuli to a higher percentage (66\%) outside of the receptive field in contrast to -1 oct (96\%, Figure 3D). Asymmetrically $\mathrm{V}$-shaped tuning curves are a consequence of cochlear mechanics
(Zwislocki, 1983; Kössl et al., 2003; Vater and Kössl, 2011) and found in many species at the cortical level (Kanwal et al., 1999; Foeller et al., 2001; Polley et al., 2007; Hoffmann et al., 2008). In addition, cortical tuning curves are modified by interaction and convergence of afferent, local, and long-range intracortical inputs (Happel et al., 2010) and different synaptic populations could be active during shaping of the respective flanks of receptive fields. As intracortical feed-forward inhibitory circuits were recently suggested to be responsible for lateral sharpening of spectral tuning (Kanwal et al., 1999; Wu et al., 2008), the high frequency flank could be more affected by this circuit activity leading to a sharper flank and thus to different CSD patterns. A relatively clear evidence for asymmetrical processing of spectrally distant stimuli of the same distance to the CF ( \pm 1 octave) can be provided by the relatively reliable appearance (68\%) of an additional late sink s9 in +loct patterns (Figure 5). The fact that $s 9$ can also be elicited with a low probability of $\sim 20 \%$ with the remaining stimuli suggests that a basic cortical pathway is involved which enhances the appearance of $s 9$ in response to certain stimuli.

Taking the cortical wiring (Figure 9) and the large onset latency distance to initial sinks $(\sim 400 \mathrm{~ms})$ into account (Figure 8), s9 is probably generated by different areas such as the FC, EC or the contralateral hemisphere. Reasons why frequencies one octave above the CF would evoke s9 remain speculative. But for the perception of communication calls which commonly consist of several harmonics (Medvedev and Kanwal, 2008; Kobayasi et al., 2012) a sink coding for higher frequencies would be of behavioral advantage as it could provide additional activity alongside s5 in layer $\mathrm{V}$ leading to subsequent behavior related areas being activated over a longer time period. The extent of synaptic activity was significantly reduced for CF24+ stimuli compared to CF80 while onset latency depth remained unchanged (Figure 8). The observed delayed onset latency for CF24+ could have another origin which is probably related to the temporal integration of the pressure envelope of the sound in the auditory periphery (Heil and Neubauer, 2001, 2003; Heil et al., 2008). These auditory nerve results were all based on neuronal spike data. Most of the sinks (Figure 6) elicited by CF24+ stimulation showed significantly reduced neuronal activity and longer onset latencies in comparison to higher level stimulation which is in accordance with a previous study concerning initial sinks (Lakatos et al., 2007). The maximum strength, onset latency, and vertical extent of s5 in layer $\mathrm{V}$ were unaffected by both spectral distance and level of the stimuli. Layer $\mathrm{V}$ integrates inputs from different cortical areas and projects back to the thalamus (Figure 9) and contains a high concentration of D1 receptors, which were suggested to modulate memory formation (Schicknick et al., 2008; Scheich et al., 2011). This could imply that s5 is a result of general modulatory actions. Interestingly, each of the mid and late sinks evoked by CF24+ was less reliably evoked than during high level stimulation. And sink s7 which during high level stimulation showed one of the largest deviations in terms of onset latency and depth (Figure 8) is quite reliably missing for CF24+ stimuli (61\%). This emphasizes that the appearance of s7 as well as the remaining mid and late sinks are dependent on the level of stimulus. 


\section{Concluding Remarks}

Our results provide a baseline for studies where precise quantitative physiological data are needed for modeling neuronal circuits involving different subcortical, intracortical, and corticocortical areas. The stimulus-specific differences in the sink metrics as well as the missing sink s7 and s9 support the hypothesis that the auditory cortex processes stimuli differently according to their frequency and level content. This could originate from stimulus-specific proportioned spatial and temporal interactions and convergences potentially leading to different processing patterns. Comparing the depth aligned onset latencies, which provide information about the sink specific laminar origins of the initial relay clusters (Figure 8), sinks s2 and s6 as well as s1 and s8, s3 and s7 or s5 and s9 (referring to s9 elicited by +loct as the others stimuli provide an insufficient data situation) could originate from common neuronal clusters. This would mean that stimulus processing is maintained by an activity

\section{References}

Ahmed, B., Anderson, J. C., Martin, K. A., and Nelson, J. C. (1997). Map of the synapses onto layer 4 basket cells of the primary visual cortex of the cat. J. Comp. Neurol. 380, 230-242. doi: 10.1002/(sici)1096-9861(19970407)380:2<230::aidcne6>3.0.co;2-4

Armstrong-James, M., and George, M. J. (1988). Influence of anesthesia on spontaneous activity and receptive field size of single units in rat Sm1 neocortex. Exp. Neurol. 99, 369-387. doi: 10.1016/0014-4886(88)90155-0

Atencio, C. A., and Schreiner, C. E. (2010). Columnar connectivity and laminar processing in cat primary auditory cortex. PLoS One 5:e9521. doi: 10. 1371/journal.pone.0009521

Barth, D. S., and Di, S. (1990). Three-dimensional analysis of auditory-evoked potentials in rat neocortex. J. Neurophysiol. 64, 1527-1536.

Barth, D. S., and Di, S. (1991). The functional anatomy of middle latency auditory evoked potentials. Brain Res. 565, 109-115. doi: 10.1016/0006-8993(91) 91741-i

Budinger, E., Heil, P., and Scheich, H. (2000). Functional organization of auditory cortex in the mongolian gerbil (Meriones unguiculatus). III. Anatomical subdivisions and corticocortical connections. Eur. J. Neurosci. 12, 2425-2451. doi: 10.1046/j.1460-9568.2000.00142.x

Budinger, E., Laszcz, A., Lison, H., Scheich, H., and Ohl, F. W. (2008). Nonsensory cortical and subcortical connections of the primary auditory cortex in Mongolian gerbils: bottom-up and top-down processing of neuronal information via field AI. Brain Res. 1220, 2-32. doi: 10.1016/j.brainres.2007. 07.084

Buser, P., and Horvath, F. E. (1972). Thalamo-caudate-cortical relationships in synchronized activity. II. Further differentiation between spindle systems by cooling and lesions in the mesencephalon. Brain Res. 39, 43-60. doi: 10. 1016/0006-8993(72)90784-6

Dantzker, J. L., and Callaway, E. M. (2000). Laminar sources of synaptic input to cortical inhibitory interneurons and pyramidal neurons. Nat. Neurosci. 3, 701-707. doi: 10.1038/76656

Di, S., and Barth, D. S. (1992). The functional anatomy of middle-latency auditory evoked potentials: thalamocortical connections. J. Neurophysiol. 68, 425-431.

Di, S., Baumgartner, C., and Barth, D. S. (1990). Laminar analysis of extracellular field potentials in rat vibrissa/barrel cortex. J. Neurophysiol. 63, 832-840.

Douglas, R. J., and Martin, K. A. (2007). Recurrent neuronal circuits in the neocortex. Curr. Biol. 17, R496-R500. doi: 10.1016/j.cub.2007.04.024

Ferreyra-Moyano, H., Cinelli, A. R., Molina, J. C., and Barragán, E. (1988). Current generators and properties of late components evoked in rat olfactory cortex. Brain Res. Bull. 20, 433-446. doi: 10.1016/0361-9230(88) 90133-5 of five main relay stations within layers I, III, Va, VIa, and VIb. Future studies on mid and late evoked sinks should focus on these layers for cortical silencing or electrical stimulation to learn more about their origins and processing mechanisms.

\section{Acknowledgments}

The authors thank Alexander Lehmann for technical support, Gisa Prange for histological support, and Jerome Beetz for useful comments to improve the science in this paper. This work was supported by the Hans-Böckler-Stiftung (Stipendium to MS).

\section{Supplementary Material}

The Supplementary Material for this article can be found online at: http://journal.frontiersin.org/article/10.3389/fncir.2015.0005 2/abstract

Foeller, E., Vater, M., and Kössl, M. (2001). Laminar analysis of inhibition in the gerbil primary auditory cortex. J. Assoc. Res. Otolaryngol. 2, 279-296. doi: 10. $1007 /$ s101620010069

Fox, S. (1970). Evoked Potential, Coding and Behavior. New York: Rockefeller University Press.

Fuster, J. M. (2000). Executive frontal functions. Exp. Brain Res. 133, 66-70. doi: 10.1007/978-3-642-59794-7_8

Fuster, J. M., and Docter, R. F. (1962). Variations of optic evoked potentials as a function of reticular activity in rabbits with chronically implanted electrodes. $J$. Neurophysiol. 25, 324-336.

Gaese, B. H., and Ostwald, J. (2001). Anesthesia changes frequency tuning of neurons in the rat primary auditory cortex. J. Neurophysiol. 86, 10621066.

Galvan, V. V., Chen, J., and Weinberger, N. M. (2002). Differential thresholds of local field potentials and unit discharges in rat auditory cortex. Hear. Res. 167, 57-60. doi: 10.1016/s0378-5955(02)00333-7

Guo, F., Intskirveli, I., Blake, D. T., and Metherate, R. (2013). Tone-detection training enhances spectral integration mediated by intracortical pathways in primary auditory cortex. Neurobiol. Learn. Mem. 101, 75-84. doi: 10.1016/j. nlm.2013.01.006

Hagemann, C., Vater, M., and Kössl, M. (2010). Comparison of properties of cortical echo delay-tuning in the short-tailed fruit bat and the mustached bat. $J$. Comp. Physiol. A Neuroethol. Sens. Neural Behav. Physiol. 197, 605-613. doi: 10. 1007/s00359-010-0530-8

Halgren, E., Marinkovic, K., and Chauvel, P. (1998). Generators of the late cognitive potentials in auditory and visual oddball tasks. Electroencephalogr. Clin. Neurophysiol. 106, 156-164. doi: 10.1016/s0013-4694(97)00119-3

Happel, M. F., Jeschke, M., and Ohl, F. W. (2010). Spectral integration in primary auditory cortex attributable to temporally precise convergence of thalamocortical and intracortical input. J. Neurosci. 30, 11114-11127. doi: 10. 1523/JNEUROSCI.0689-10.2010

Hechavarria, J. C., Macias, S., Vater, M., Voss, C., Mora, E. C., and Kössl, M. (2013). Blurry topography for precise target-distance computations in the auditory cortex of echolocating bats. Nat. Commun. 4:2587. doi: 10 . $1038 /$ ncomms3587

Heil, P., and Neubauer, H. (2001). Temporal integration of sound pressure determines thresholds of auditory-nerve fibers. J. Neurosci. 21, 74047415

Heil, P., and Neubauer, H. (2003). A unifying basis of auditory thresholds based on temporal summation. Proc. Natl. Acad. Sci. U S A 100, 6151-6156. doi: 10. 1073/pnas.1030017100

Heil, P., Neubauer, H., Brown, M., and Irvine, D. R. (2008). Towards a unifying basis of auditory thresholds: distributions of the first-spike latencies 
of auditory-nerve fibers. Hear. Res. 238, 25-38. doi: 10.1016/j.heares.2007. 09.014

Hoffmann, S., Firzlaff, U., Radtke-Schuller, S., Schwellnus, B., and Schuller, G. (2008). The auditory cortex of the bat Phyllostomus discolor: localization and organization of basic response properties. BMC Neurosci. 9:65. doi: 10. 1186/1471-2202-9-65

Horvath, F. E., and Buser, P. (1972). Thalamo-caudate-cortical relationships in synchronized activity. I. Differentiation between ventral and dorsal spindle systems. Brain Res. 39, 21-41. doi: 10.1016/0006-8993(72) 90783-4

Huang, C. L., and Winer, J. A. (2000). Auditory thalamocortical projections in the cat: laminar and areal patterns of input. J. Comp. Neurol. 427, 302-331. doi: 10. 1002/1096-9861(20001113)427:2<302::aid-cne10>3.0.co;2-j

Insausti, R., Herrero, M. T., and Witter, M. P. (1997). Entorhinal cortex of the rat: cytoarchitectonic subdivisions and the origin and distribution of cortical efferents. Hippocampus 7, 146-183. doi: 10.1002/(sici)10981063(1997)7:2<146::aid-hipo4>3.0.co;2-1

Iwasa, H., and Potsic, W. P. (1982). Maturational change of early, middle and late components of the auditory evoked responses in rats. Otolaryngol. Head Neck Surg. 90, 95-102.

Izhikevich, E. M., and Edelman, G. M. (2008). Large-scale model of mammalian thalamocortical systems. Proc. Natl. Acad. Sci. U S A 105, 3593-3598. doi: 10. 1073/pnas.0712231105

Jacobs, J., Kahana, M. J., Ekstrom, A. D., Mollison, M. V., and Fried, I. (2010). A sense of direction in human entorhinal cortex. Proc. Natl. Acad. Sci. U S A 107, 6487-6492. doi: 10.1073/pnas.0911213107

John, E. (1967). Electrophysiological Studies of Conditioning. New York: Rockefeller University Press.

Kaga, K., Hink, R. F., Shinoda, Y., and Suzuki, J. (1980). Evidence for a primary cortical origin of a middle latency auditory evoked potential in cats. Electroencephalogr. Clin. Neurophysiol. 50, 254-266. doi: 10.1016/00134694(80)90153-4

Kajikawa, Y., and Schroeder, C. E. (2011). How local is the local field potential? Neuron 72, 847-858. doi: 10.1016/j.neuron.2011.09.029

Kanwal, J. S., Fitzpatrick, D. C., and Suga, N. (1999). Facilitatory and inhibitory frequency tuning of combination-sensitive neurons in the primary auditory cortex of mustached bats. J. Neurophysiol. 82, 2327-2345.

Kanwal, J. S., Gordon, M., Peng, J. P., and Heinz-Esser, K. (2000). Auditory responses from the frontal cortex in the mustached bat, Pteronotus parnellii. Neuroreport 11, 367-372. doi: 10.1097/00001756-20000207000029

Kaur, S., Lazar, R., and Metherate, R. (2004). Intracortical pathways determine breadth of subthreshold frequency receptive fields in primary auditory cortex. J. Neurophysiol. 91, 2551-2567. doi: 10.1152/jn.01121.2003

Kaur, S., Rose, H. J., Lazar, R., Liang, K., and Metherate, R. (2005). Spectral integration in primary auditory cortex: laminar processing of afferent input, in vivo and in vitro. Neuroscience 134, 1033-1045. doi: 10.1016/j.neuroscience. 2005.04.052

Kimura, A., Donishi, T., Sakoda, T., Hazama, M., and Tamai, Y. (2003). Auditory thalamic nuclei projections to the temporal cortex in the rat. Neuroscience 117, 1003-1016. doi: 10.1016/s0306-4522(02)00949-1

Knight, R. T., Brailowsky, S., Scabini, D., and Simpson, G. V. (1985). Surface auditory evoked potentials in the unrestrained rat: component definition. Electroencephalogr. Clin. Neurophysiol. 61, 430-439. doi: 10.1016/00134694(85)91035-1

Kobayasi, K. I., Usami, A., and Riquimaroux, H. (2012). Behavioral evidence for auditory induction in a species of rodent: Mongolian gerbil (Meriones unguiculatus). J. Acoust. Soc. Am. 132, 4063-4068. doi: 10.1121/1.4763546

Kosel, K. C., Van Hoesen, G. W., and Rosene, D. L. (1982). Non-hippocampal cortical projections from the entorhinal cortex in the rat and rhesus monkey. Brain Res. 244, 201-213. doi: 10.1016/0006-8993(82)90079-8

Kössl, M., Foeller, E., Drexl, M., Vater, M., Mora, E., Coro, F., et al. (2003). Postnatal development of cochlear function in the mustached bat, Pteronotus parnellii. J. Neurophysiol. 90, 2261-2273. doi: 10.1152/jn.00 100.2003

Kraus, N., Smith, D. I., and Grossmann, J. (1985). Cortical mapping of the auditory middle latency response in the unanesthetized guinea pig. Electroencephalogr. Clin. Neurophysiol. 62, 219-226. doi: 10.1016/0168-5597(85)90017-6
Kraut, M. A., Arezzo, J. C., and Vaughan, H. G. Jr. (1985). Intracortical generators of the flash VEP in monkeys. Electroencephalogr. Clin. Neurophysiol. 62, 300-312. doi: 10.1016/0168-5597(85)90007-3

Kulics, A. T., and Cauller, L. J. (1986). Cerebral cortical somatosensory evoked-responses, multiple unit-activity and current source-densities - their interrelationships and significance to somatic sensation as revealed by stimulation of the awake monkeys hand. Exp. Brain Res. 62, 46-60. doi: 10 1007/bf00237402

Kurt, S., Deutscher, A., Crook, J. M., Ohl, F. W., Budinger, E., Moeller, C. K., et al. (2008). Auditory cortical contrast enhancing by global winner-take-all inhibitory interactions. PLoS One 3:e1735. doi: 10.1371/journal.pone.0001735

Kuwada, S., Batra, R., and Stanford, T. R. (1989). Monaural and binaural response properties of neurons in the inferior colliculus of the rabbit: effects of sodium pentobarbital. J. Neurophysiol. 61, 269-282.

Lakatos, P., Chen, C. M., O'Connell, M. N., Mills, A., and Schroeder, C. E. (2007). Neuronal oscillations and multisensory interaction in primary auditory cortex. Neuron 53, 279-292. doi: 10.1016/j.neuron.2006.12.011

Lakatos, P., O'Connell, M. N., Barczak, A., Mills, A., Javitt, D. C., and Schroeder, C. E. (2009). The leading sense: supramodal control of neurophysiological context by attention. Neuron 64, 419-430. doi: 10.1016/j.neuron.2009.10.014

Lakatos, P., Shah, A. S., Knuth, K. H., Ulbert, I., Karmos, G., and Schroeder, C. E. (2005). An oscillatory hierarchy controlling neuronal excitability and stimulus processing in the auditory cortex. J. Neurophysiol. 94, 1904-1911. doi: 10. $1152 /$ jn. 00263.2005

Lee, C. C., and Winer, J. A. (2008). Connections of cat auditory cortex: I. Thalamocortical system. J. Comp. Neurol. 507, 1879-1900. doi: 10.1002/cne. 21611

Linden, J. F., and Schreiner, C. E. (2003). Columnar transformations in auditory cortex? A comparison to visual and somatosensory cortices. Cereb. Cortex 13, 83-89. doi: 10.1093/cercor/13.1.83

Logothetis, N. K. (2008). What we can do and what we cannot do with fMRI. Nature 453, 869-878. doi: 10.1038/nature06976

Maier, A., Aura, C. J., and Leopold, D. A. (2011). Infragranular sources of sustained local field potential responses in macaque primary visual cortex. J. Neurosci. 31, 1971-1980. doi: 10.1523/JNEUROSCI.5300-09.2011

Matsubara, J. A., and Phillips, D. P. (1988). Intracortical connections and their physiological correlates in the primary auditory cortex (AI) of the cat. J. Comp. Neurol. 268, 38-48. doi: 10.1002/cne.902680105

Medvedev, A. V., and Kanwal, J. S. (2004). Local field potentials and spiking activity in the primary auditory cortex in response to social calls. $J$. Neurophysiol. 92, 52-65. doi: 10.1152/jn.01253.2003

Medvedev, A. V., and Kanwal, J. S. (2008). Communication call-evoked gamma-band activity in the auditory cortex of awake bats is modified by complex acoustic features. Brain Res. 1188, 76-86. doi: 10.1016/j.brainres.2007. 10.081

Mendelson, J. R., Schreiner, C. E., and Sutter, M. L. (1997). Functional topography of cat primary auditory cortex: response latencies. J. Comp. Physiol. A 181, 615-633. doi: 10.1007/s003590050145

Mitani, A., Shimokouchi, M., Itoh, K., Nomura, S., Kudo, M., and Mizuno, N. (1985). Morphology and laminar organization of electrophysiologically identified neurons in the primary auditory cortex in the cat. J. Comp. Neurol. 235, 430-447. doi: 10.1002/cne.902350403

Mitchell, B. D., and Macklis, J. D. (2005). Large-scale maintenance of dual projections by callosal and frontal cortical projection neurons in adult mice. J. Comp. Neurol. 482, 17-32. doi: 10.1002/cne.20428

Mitzdorf, U. (1985). Current source-density method and application in cat cerebral cortex: investigation of evoked potentials and EEG phenomena. Physiol. Rev. 65, 37-100.

Mitzdorf, U. (1987). Properties of the evoked potential generators: current sourcedensity analysis of visually evoked potentials in the cat cortex. Int. J. Neurosci. 33, 33-59. doi: 10.3109/00207458708985928

Mitzdorf, U., and Singer, W. (1979). Excitatory synaptic ensemble properties in the visual cortex of the macaque monkey: a current source density analysis of electrically evoked potentials. J. Comp. Neurol. 187, 71-83. doi: 10.1002/cne. 901870105

Moeller, C. K., Kurt, S., Happel, M. F., and Schulze, H. (2010). Long-range effects of GABAergic inhibition in gerbil primary auditory cortex. Eur. J. Neurosci. 31, 49-59. doi: 10.1111/j.1460-9568.2009.07039.x 
Müller-Preuss, P., and Mitzdorf, U. (1984). Functional anatomy of the inferior colliculus and the auditory cortex: current source density analyses of clickevoked potentials. Hear. Res. 16, 133-142. doi: 10.1016/0378-5955(84)90003-0

Näätänen, R., and Picton, T. (1987). The N1 wave of the human electric and magnetic response to sound: a review and an analysis of the component structure. Psychophysiology 24, 375-425. doi: 10.1111/j.1469-8986. 1987.tb00311.x

Nicholson, C., and Freeman, J. A. (1975). Theory of current source-density analysis and determination of conductivity tensor for anuran cerebellum. J. Neurophysiol. 38, 356-368. doi: 10.1016/0006-8993(75)90494-1

O’Connell, M. N., Falchier, A., McGinnis, T., Schroeder, C. E., and Lakatos, P. (2011). Dual mechanism of neuronal ensemble inhibition in primary auditory cortex. Neuron 69, 805-817. doi: 10.1016/j.neuron.2011.01.012

Ohl, F. W., Scheich, H., and Freeman, W. J. (2000). Topographic analysis of epidural pure-tone-evoked potentials in gerbil auditory cortex. J. Neurophysiol. $83,3123-3132$.

Ojima, H., Honda, C. N., and Jones, E. G. (1991). Patterns of axon collateralization of identified supragranular pyramidal neurons in the cat auditory cortex. Cereb. Cortex 1, 80-94. doi: 10.1093/cercor/1.1.80

Ojima, H., Honda, C. N., and Jones, E. G. (1992). Characteristics of intracellularly injected infragranular pyramidal neurons in cat primary auditory cortex. Cereb. Cortex 2, 197-216. doi: 10.1093/cercor/2.3.197

Peters, A., Payne, B. R., and Budd, J. (1994). A numerical analysis of the geniculocortical input to striate cortex in the monkey. Cereb. Cortex 4, 215-229. doi: 10.1093/cercor/4.3.215

Petersen, C. C., Hahn, T. T., Mehta, M., Grinvald, A., and Sakmann, B. (2003). Interaction of sensory responses with spontaneous depolarization in layer $2 / 3$ barrel cortex. Proc. Natl. Acad. Sci. U S A 100, 13638-13643. doi: 10.1073/pnas. 2235811100

Pettersen, K. H., Devor, A., Ulbert, I., Dale, A. M., and Einevoll, G. T. (2006). Current-source density estimation based on inversion of electrostatic forward solution: effects of finite extent of neuronal activity and conductivity discontinuities. J. Neurosci. Methods 154, 116-133. doi: 10.1016/j.jneumeth. 2005.12.005

Phillips, D. P., and Irvine, D. R. (1981). Responses of single neurons in physiologically defined primary auditory cortex (AI) of the cat: frequency tuning and responses to intensity. J. Neurophysiol. 45, 48-58.

Phillips, D. P., Orman, S. S., Musicant, A. D., and Wilson, G. F. (1985). Neurons in the cat's primary auditory cortex distinguished by their responses to tones and wide-spectrum noise. Hear. Res. 18, 73-86. doi: 10.1016/0378-5955(85) 90111-x

Picton, T. W., Hillyard, S. A., Krausz, H. I., and Galambos, R. (1974). Human auditory evoked potentials. I. Evaluation of components. Electroencephalogr. Clin. Neurophysiol. 36, 179-190. doi: 10.1016/0013-4694(74)90155-2

Pockberger, H., and Rappelsberger, P. (1983). Mechanism of the generation of evoked potentials in the rabbit neocortex. EEG EMG Z. Elektroenzephalogr. Elektromyogr. Verwandte Geb. 14, 169-176.

Polich, J., Aung, M., and Dalessio, D. J. (1988). Long latency auditory evoked potentials: intensity, inter-stimulus interval and habituation. Pavlov. J. Biol. Sci. 23, 35-40.

Polley, D. B., Read, H. L., Storace, D. A., and Merzenich, M. M. (2007). Multiparametric auditory receptive field organization across five cortical fields in the albino rat. J. Neurophysiol. 97, 3621-3638. doi: 10.1152/jn.012 98.2006

Rappelsberger, P., Pockberger, H., and Petsche, H. (1981). Current source density analysis: methods and application to simultaneously recorded field potentials of the rabbit's visual cortex. Pflugers Arch. 389, 159-170. doi: 10.1007/bf005 82108

Read, H. L., Winer, J. A., and Schreiner, C. E. (2001). Modular organization of intrinsic connections associated with spectral tuning in cat auditory cortex. Proc. Natl. Acad. Sci. U S A 98, 8042-8047. doi: 10.1073/pnas.131591898

Ryan, A. (1976). Hearing sensitivity of the mongolian gerbil, meriones unguiculatis. J. Acoust. Soc. Am. 59, 1222-1226. doi: 10.1121/1.380961

Sakamoto, K., Kawaguchi, N., Yagi, K., and Mushiake, H. (2015). Spatiotemporal patterns of current source density in the prefrontal cortex of a behaving monkey. Neural Netw. 62, 67-72. doi: 10.1016/j.neunet.2014.06.009

Sakhiulina, G. T., and Merzhanova, G. K. (1966). Stable changes in the pattern of the recruiting response associated with a well established conditioned reflex. Electroencephalogr. Clin. Neurophysiol. 20, 50-58. doi: 10.1016/00134694(66)90140-4

Saleem, A. B., Chadderton, P., Apergis-Schoute, J., Harris, K. D., and Schultz, S. R. (2010). Methods for predicting cortical UP and DOWN states from the phase of deep layer local field potentials. J. Comput. Neurosci. 29, 49-62. doi: 10.3410/f. 3184978.2877063

Santos Filha, V. A. V. D., and Matas, C. G. (2010). Late Auditory evoked potentials in individuals with tinnitus. Braz. J. Otorhinolaryngol. 76, 263-270. doi: 10. 1590/S1808-86942010000200019

Satya-Murti, S., Wolpaw, J. R., Cacace, A. T., and Schaffer, C. A. (1983). Late auditory evoked potentials can occur without brain stem potentials. Electroencephalogr. Clin. Neurophysiol. 56, 304-308. doi: 10.1016/00134694(83)90255-9

Scheich, H., Brechmann, A., Brosch, M., Budinger, E., Ohl, F. W., Selezneva, E., et al. (2011). Behavioral semantics of learning and crossmodal processing in auditory cortex: the semantic processor concept. Hear. Res. 271, 3-15. doi: 10. 1016/j.heares.2010.10.006

Schicknick, H., Schott, B. H., Budinger, E., Smalla, K. H., Riedel, A., Seidenbecher, C. I., et al. (2008). Dopaminergic modulation of auditory cortex-dependent memory consolidation through mTOR. Cereb. Cortex 18, 2646-2658. doi: 10. 1093/cercor/bhn026

Schroeder, C. E., Tenke, C. E., Givre, S. J., Arezzo, J. C., and Vaughan, H. G. Jr. (1990). Laminar analysis of bicuculline-induced epileptiform activity in area 17 of the awake macaque. Brain Res. 515, 326-330. doi: 10.1016/00068993(90)90617-k

Shen, J. X., Xu, Z. M., and Yao, Y. D. (1999). Evidence for columnar organization in the auditory cortex of the mouse. Hear. Res. 137, 174-177. doi: 10.1016/s03785955(99)00149-5

Shepherd, G., and Koch, C. (1998). Introduction to Synaptic Circuits. New York: Oxford University Press.

Simpson, G. V., and Knight, R. T. (1993). Multiple brain systems generating the rat auditory evoked potential. I. Characterization of the auditory cortex response. Brain Res. 602, 240-250. doi: 10.1016/0006-8993(93)90689-k

Skrebitsky, V. G., and Sharonova, I. N. (1976). Reticular suppression of flashevoked IPSPs in visual cortex neurons. Brain Res. 111, 67-78. doi: 10 1016/0006-8993(76)91049-0

Sloan, T. B. (1998). Anesthetic effects on electrophysiologic recordings. J. Clin. Neurophysiol. 15, 217-226. doi: 10.1097/00004691-199805000-00005

Steinschneider, M., Reser, D. H., Fishman, Y. I., Schroeder, C. E., and Arezzo, J. C. (1998). Click train encoding in primary auditory cortex of the awake monkey: evidence for two mechanisms subserving pitch perception. J. Acoust. Soc. Am. 104, 2935-2955. doi: 10.1121/1.423877

Steinschneider, M., Tenke, C. E., Schroeder, C. E., Javitt, D. C., Simpson, G. V., Arezzo, J. C., et al. (1992). Cellular generators of the cortical auditory evoked potential initial component. Electroencephalogr. Clin. Neurophysiol. 84, 196-200. doi: 10.1016/0168-5597(92)90026-8

Sugimoto, S., Sakurada, M., Horikawa, J., and Taniguchi, I. (1997). The columnar and layer-specific response properties of neurons in the primary auditory cortex of Mongolian gerbils. Hear. Res. 112, 175-185. doi: 10.1016/s03785955(97)00119-6

Szymanski, F. D., Garcia-Lazaro, J. A., and Schnupp, J. W. (2009). Current source density profiles of stimulus-specific adaptation in rat auditory cortex. J. Neurophysiol. 102, 1483-1490. doi: 10.1152/jn.00240.2009

Takahashi, H., Nakao, M., and Kaga, K. (2007). Multiple neural origins of early auditory evoked potential of rat. Neuroscience 148, 845-856. doi: 10.1016/j. neuroscience.2007.07.023

Tenke, C. E., and Kayser, J. (2012). Generator localization by current source density (CSD): implications of volume conduction and field closure at intracranial and scalp resolutions. Clin. Neurophysiol. 123, 2328-2345. doi: 10. 1016/j.clinph.2012.06.005

Tenke, C. E., Schroeder, C. E., Arezzo, J. C., and Vaughan, H. G. Jr. (1993). Interpretation of high-resolution current source density profiles: a simulation of sublaminar contributions to the visual evoked potential. Exp. Brain Res. 94, 183-192. doi: 10.1007/bf00230286

Thomas, H., Tillein, J., Heil, P., and Scheich, H. (1993). Functional organization of auditory cortex in the mongolian gerbil (Meriones unguiculatus). I. Electrophysiological mapping of frequency representation and distinction of fields. Eur. J. Neurosci. 5, 882-897. doi: 10.1111/j.1460-9568.1993.tb00940.x 
Thomson, A. M., and Bannister, A. P. (2003). Interlaminar connections in the neocortex. Cereb. Cortex 13, 5-14. doi: 10.1093/cercor/13.1.5

Thomson, A. M., and Lamy, C. (2007). Functional maps of neocortical local circuitry. Front. Neurosci. 1, 19-42. doi: 10.3389/neuro.01.1.1.002.2007

Timofeev, I., and Steriade, M. (1998). Cellular mechanisms underlying intrathalamic augmenting responses of reticular and relay neurons. J. Neurophysiol. 79, 2716-2729.

Tomioka, R., Okamoto, K., Furuta, T., Fujiyama, F., Iwasato, T., Yanagawa, Y., et al. (2005). Demonstration of long-range GABAergic connections distributed throughout the mouse neocortex. Eur. J. Neurosci. 21, 1587-1600. doi: 10. 1111/j.1460-9568.2005.03989.x

Torres, F., and Warner, J. S. (1962). Some characteristics of delayed responses to photic stimuli in the cat. Electroencephalogr. Clin. Neurophysiol. 14, 654-663. doi: 10.1016/0013-4694(62)90080-9

Vaknin, G., Discenna, P. G., and Teyler, T. J. (1988). A method for calculating current source density (CSD) analysis without resorting to recording sites outside the sampling volume. J. Neurosci. Methods 24, 131-135. doi: 10. 1016/0165-0270(88)90056-8

Vater, M., and Kössl, M. (2011). Comparative aspects of cochlear functional organization in mammals. Hear. Res. 273, 89-99. doi: 10.1016/j.heares.2010. 05.018

Wachtmeister, L., and Dowling, J. E. (1978). The oscillatory potentials of the mudpuppy retina. Invest. Ophthalmol. Vis. Sci. 17, 1176-1188.

Wallace, M. N., and Palmer, A. R. (2008). Laminar differences in the response properties of cells in the primary auditory cortex. Exp. Brain Res. 184, 179-191. doi: $10.1007 / \mathrm{s} 00221-007-1092-\mathrm{z}$
Wessinger, C. M., Vanmeter, J., Tian, B., Van Lare, J., Pekar, J., and Rauschecker, J. P. (2001). Hierarchical organization of the human auditory cortex revealed by functional magnetic resonance imaging. J. Cogn. Neurosci. 13, 1-7. doi: 10. $1162 / 089892901564108$

Winer, J. A., and Lee, C. C. (2007). The distributed auditory cortex. Hear. Res. 229, 3-13. doi: 10.1016/j.heares.2007.01.017

Wu, G. K., Arbuckle, R., Liu, B. H., Tao, H. W., and Zhang, L. I. (2008). Lateral sharpening of cortical frequency tuning by approximately balanced inhibition. Neuron 58, 132-143. doi: 10.1016/j.neuron.2008.01.035

Zurita, P., Villa, A. E., de Ribaupierre, Y., de Ribaupierre, F., and Rouiller, E. M. (1994). Changes of single unit activity in the cat's auditory thalamus and cortex associated to different anesthetic conditions. Neurosci. Res. 19, 303-316. doi: 10. 1016/0168-0102(94)90043-4

Zwislocki, J. J. (1983). Some current concepts of cochlear mechanics. Audiology 22, 517-529. doi: 10.3109/00206098309072811

Conflict of Interest Statement: The authors declare that the research was conducted in the absence of any commercial or financial relationships that could be construed as a potential conflict of interest.

Copyright (c) 2015 Schaefer, Hechavarría and Kössl. This is an open-access article distributed under the terms of the Creative Commons Attribution License (CC BY). The use, distribution and reproduction in other forums is permitted, provided the original author(s) or licensor are credited and that the original publication in this journal is cited, in accordance with accepted academic practice. No use, distribution or reproduction is permitted which does not comply with these terms. 